\title{
The survey of planetary nebulae in Andromeda (M 31)
}

\section{Imaging the disc and halo with MegaCam at the CFHT^}

\author{
Souradeep Bhattacharya (মৌরদীপ ভট্টাচার্য্য) ${ }^{1}$, Magda Arnaboldi ${ }^{1}$, Johanna Hartke ${ }^{1,2}$, Ortwin Gerhard ${ }^{3}$, \\ Valentin Comte ${ }^{1,4}$, Alan McConnachie ${ }^{5}$, and Nelson Caldwell ${ }^{6}$
}

\author{
${ }^{1}$ European Southern Observatory, Karl-Schwarzschild-Str. 2, 85748 Garching, Germany \\ e-mail: sbhattac@eso.org \\ 2 European Southern Observatory, Alonso de Cordova 3107, Vitacura, Casilla 19001, Santiago de Chile, Chile \\ 3 Max-Planck-Institut für Extraterrestrische Physik, Giessenbachstrasse, 85748 Garching, Germany \\ 4 Aix Marseille Université, CNRS, LAM-Laboratoire d'Astrophysique de Marseille, 38 rue F. Joliot-Curie, 13388 Marseille, \\ France \\ 5 NRC Herzberg Institute of Astrophysics, 5071 West Saanich Road, Victoria, BC V9E 2E7, Canada \\ ${ }^{6}$ Harvard-Smithsonian Center for Astrophysics, 60 Garden Street, Cambridge, MA 02138, USA
}

Received 5 November 2018 / Accepted 6 March 2019

\begin{abstract}
Context. The Andromeda (M 31) galaxy subtends nearly 100 square degrees on the sky. Any study of its halo must therefore account for the severe contamination from the Milky Way halo stars whose surface density displays a steep gradient across the entire M 31 field of view.

Aims. Our goal is to identify a population of stars firmly associated with the M 31 galaxy. Planetary nebulae (PNe) are one such population that are excellent tracers of light, chemistry, and motion in galaxies. We present a 16 square degree survey of the disc and inner halo of M31 with the MegaCam wide-field imager at the CFHT to identify PNe, and characterise the luminosity-specific PN number and PN luminosity function (PNLF) in M31.

Methods. PNe were identified via automated detection techniques based on their bright [O III] $5007 \AA$ emission and absence of a continuum. Subsamples of the faint PNe were independently confirmed by matching with resolved Hubble Space Telescope sources from the Panchromatic Hubble Andromeda Treasury and spectroscopic follow-up observations with HectoSpec at the MMT.

Results. The current survey reaches two magnitudes fainter than the previous most sensitive survey. We thus identify $4289 \mathrm{PNe}$, of which only 1099 were previously known. By comparing the PN number density with the surface brightness profile of M 31 out to $\sim 30 \mathrm{kpc}$ along the minor axis, we find that the stellar population in the inner halo has a luminosity-specific PN number value that is seven times higher than that of the disc. We measure the luminosity function of the PN population and find a bright cut-off and a slope consistent with previous determinations. Interestingly, it shows a significant rise at the faint end, present in all radial bins covered by the survey. This rise in the M31 PNLF is much steeper than that observed for the Magellanic clouds and Milky Way bulge.

Conclusions. The significant radial variation of the PN specific frequency value indicates that the stellar population at deprojected minor-axis radii larger than $\sim 10 \mathrm{kpc}$ is different from that in the disc of M31. The rise at the faint end of the PNLF is a property of the late phases of the stellar population. M 31 shows two major episodes of star formation and the rise at the faint end of the PNLF is possibly associated with the older stellar population. It may also be a result of varying opacity of the PNe.
\end{abstract}

Key words. galaxies: individual: M31 - galaxies: halos - planetary nebulae: general

\section{Introduction}

The Andromeda galaxy, M31, is the closest giant spiral disc to our Milky Way (MW). It lies at a distance of $\sim 780 \mathrm{kpc}$ with a high inclination to the line of sight $(i \sim 77 \mathrm{deg})$ making it ideally suited for studies of its halo regions. Since galaxies are believed to be formed by hierarchical mass assembly, their outskirts with long dynamical timescales

* Based on observations obtained with MegaCam, a joint project of the CFHT and CEA/DAPNIA, at the Canada-France-Hawaii Telescope (CFHT), which is operated by the National Research Council (NRC) of Canada, the Institut National des Sciences de l'Univers of the Centre National de la Recherche Scientifique (CNRS) of France, and the University of Hawaii. The observations at the CFHT were performed with care and respect from the summit of Maunakea which is a significant cultural and historic site. are expected to have coherent debris from past accretion events for the greatest longevity (Ferguson \& Mackey 2016). Through the Pan-Andromeda Archaeological Survey (PAndAS; McConnachie et al. 2009) map of the resolved stellar population number counts, we now know about the substructures present in the M 31 halo (Giant Stellar Stream, G1 and NE clump, NE and W shelves; McConnachie et al. 2018) and that the stellar halo extends out to $165 \mathrm{kpc}$.

Because of the faint surface brightness of the M31 halo, $\mu_{v}>25$ outside the main disc ( $\sim 15 \mathrm{kpc}$ major-axis distance), the use of discrete stellar tracers is superior to integrated absorptionline spectroscopy in providing a global mapping of the halo kinematics. Globular clusters (GCs) have been shown to efficiently trace the outer halo (outside $50 \mathrm{kpc}$ ) substructures of M31 (Mackey et al. 2010; Veljanoski et al. 2014) bolstering the idea that these clusters and the substructures they trace have been 
accreted in various merger events. However, the inner halo substructures (within $50 \mathrm{kpc}$ ) are not well traced by the GCs. Some of these substructures, especially the Giant Stream, may have resulted from a single merger event $\sim 2 \mathrm{Gyr}$ ago (Bernard et al. 2015; Hammer et al. 2018) perhaps between M 31 and the large $\left(M_{\mathrm{M} 32 \mathrm{P}} \sim 2.5 \times 10^{10} M_{\odot}\right)$ progenitor of M 32 (D'Souza \& Bell 2018).

We can gain information on the motions of the stars in the low surface brightness regions of M 31 by studying planetary nebulae $(\mathrm{PNe})$ that act as discrete tracers of stars in the halo. PNe are the glowing shells of gas and dust observed around stars that have recently left the asymptotic giant branch (AGB) and are evolving towards the white dwarf stage. They are traditionally considered the late phases of stars with masses between $\sim 0.7$ and $8 M_{\odot}$, but have been shown to exhibit a wide variety of striking morphologies pointing towards a binary evolution in many systems (Jones \& Boffin 2017). Since the timescales between the AGB and PN phases are short, the distribution and kinematics of $\mathrm{PNe}$ are expected to be identical to their parent population, having the same angular momentum distribution as the stellar population (e.g., Hui et al. 1995; Arnaboldi et al. 1996, 1998; Méndez et al. 2001). Studying PNe as a population provides insight into galactic structure and evolution. Because of their relatively strong [O III] $5007 \AA$ emission, PNe can be readily identified. They have been shown to be efficient tracers of stellar light in different galaxies like M 87 (Longobardi et al. 2013), M 49 (Hartke et al. 2017), and many other early-type galaxies (Coccato et al. 2009; Cortesi et al. 2013; Pulsoni et al. 2018). The luminosity-specific PN number ( $\alpha$-parameter) varies slightly with $B-V$ colour of galaxies with higher values for late-type galaxies and lower values for early-type galaxies (Buzzoni et al. 2006). Different $\alpha$-parameter values point to differences in stellar populations even within the same galaxy (e.g., M 49; Hartke et al. 2017).

The characteristic [O III] $5007 \AA$ PN luminosity function (PNLF) has proven itself to be a reliable secondary distance indicator for determining galactic distances out to $\sim 20 \mathrm{Mpc}$ by virtue of its invariant absolute bright cut-off, $M^{*}$. The faint end of the PNLF was shown by Jacoby (1980) to follow an exponential function expected from slowly evolving central stars embedded in rapidly expanding, optically thin nebulae (Henize \& Westerlund 1963). The PNLF was first described by Ciardullo et al. (1989) estimated empirically from the brightest PN they found in the centre of M31 as

$N(M) \propto \mathrm{e}^{0.307 M}\left(1-\mathrm{e}^{3\left(M^{*}-M\right)}\right)$.

The bright end exponential cut-off, supported with accurate measures of foreground extinction, is currently measured at $M^{*}=-4.54 \pm 0.05$ (Ciardullo et al. 2013). Apart from $M^{*}$ reducing in low metallicity populations (e.g., Ciardullo \& Jacoby 1992; Ciardullo et al. 2002; Hernández-Martínez \& Peña 2009), the PNLF cut-off has proved to be largely invariant with metallicity and age of the parent stellar population, and galaxy type.

The faint end of the PNLF has been shown to vary considerably, depending on the details of the stellar population. It is seen to be correlated with the star formation history of the parent stellar population, with steeper slopes associated with older stellar populations and conversely flatter slopes with younger populations (Ciardullo et al. 2004; Ciardullo 2010; Longobardi et al. 2013; Hartke et al. 2017). The changes in the PNLF slope can result from the superposition of multiple stellar populations, which can then be disentangled using the PNe kinematics (Longobardi et al. 2015; Hartke et al. 2018). The PNLF has also been shown to display a dip for some galaxies. The dip is seen $\sim 3.5$ mag below the bright cut-off in the Small Magellanic Cloud (SMC; Jacoby \& De Marco 2002), 2.5 mag below the bright cut-off for NGC 6822 (Hernández-Martínez \& Peña 2009) and also slightly in the Large Magellanic Clouds (Reid \& Parker 2010), and $\sim 1$ mag below the bright cut-off in M 87 (Longobardi et al. 2015). This dip is thought to be related to the opacity of the PN and may be characterised by accounting for circumstellar extinction in the PNe. Circumstellar extinction correction indeed modifies the PNLF (Reid \& Parker 2010; Davis et al. 2018), but it is difficult to estimate for the faint end of the PNLF (beyond $\sim 2.5$ mag below the bright cut-off) to test for changes in opacity of the PN. Additionally, PNe mimics like $\mathrm{H}_{\text {II }}$ regions and symbiotic stars (SySts) may be misidentified as PNe thereby affecting the PNLF.

Since the first empirical study of the PNLF by Ciardullo et al. (1989) using 104 objects in the M31 bulge, the number of PNe known in M 31 has increased by leaps and bounds. Most notably, Merrett et al. (2006, hereafter M06) utilised the custom-built Planetary Nebula Spectrograph (PNS; Douglas et al. 2002) at the William Herschel Telescope (WHT) to identify $2615 \mathrm{PNe}$ in the disc and bulge of M31, and to simultaneously obtain their [O III] $5007 \AA$ magnitude and line-of-sight velocity (LOSV). M06 corroborated the PNLF found by Ciardullo et al. (1989) and increased the photometric depth to $\sim 3.5-4$ mag below the bright cut-off along with the increased uniform coverage. Since then, Martin et al. (2018) and Li et al. (2018) have identified more PNe in the central regions and circumnuclear region of M31, respectively, adding to the large number of PNe already known in M 31.

While some of the PNe identified by M06 have been shown to be H II regions (Sanders et al. 2012; Veyette et al. 2014), the M06 PNe sample remains the largest uniform sample of PNe in any galaxy. The LOSV of the M06 PNe predicted links between the NE shelf and the giant stream substructures of M 31 which have been further explored in deep spectroscopic chemical tagging studies by Fang et al. $(2015,2018)$ to further establish these links. However, while some identified PNe have been associated with the halo, a uniform survey of PNe in much of the metalpoor halo of M31 and the inner-halo substructures is necessary not only to unambiguously trace them, but also to probe the variation in the PNLF further out from the disc to corroborate the invariant nature of its bright cut-off and observe the evolutionary effects on its faint end.

In this paper, we survey the inner 16 square degrees of M 31 (corresponding to $20-30 \mathrm{kpc}$ from the centre), covering the disc, parts of the inner halo, and some of the inner halo substructures. We detect PNe using the on-off-band technique to a depth further than M06. We describe the observations and data reduction in Sect. 2, and the identification of PNe in Sect. 3. In Sect. 4 we describe the identification of the PNe counterparts in a subsample of Hubble Space Telescope (HST) data. We obtain the $\alpha$-parameter in Sect. 5 and analyse the PNLF in Sect. 6. We present the discussions of the results in Sect. 7, and we summarise and conclude in Sect. 8.

\section{CFHT MegaCam M 31 PNe survey}

\subsection{Imaging and observations}

The observations were carried out with the MegaCam widefield imager (Boulade et al. 2003) mounted on the $3.6 \mathrm{~m}$ CanadaFrance-Hawaii Telescope (CFHT), located near the summit of the Mauna Kea mountain on Hawaii's Big Island at an altitude 


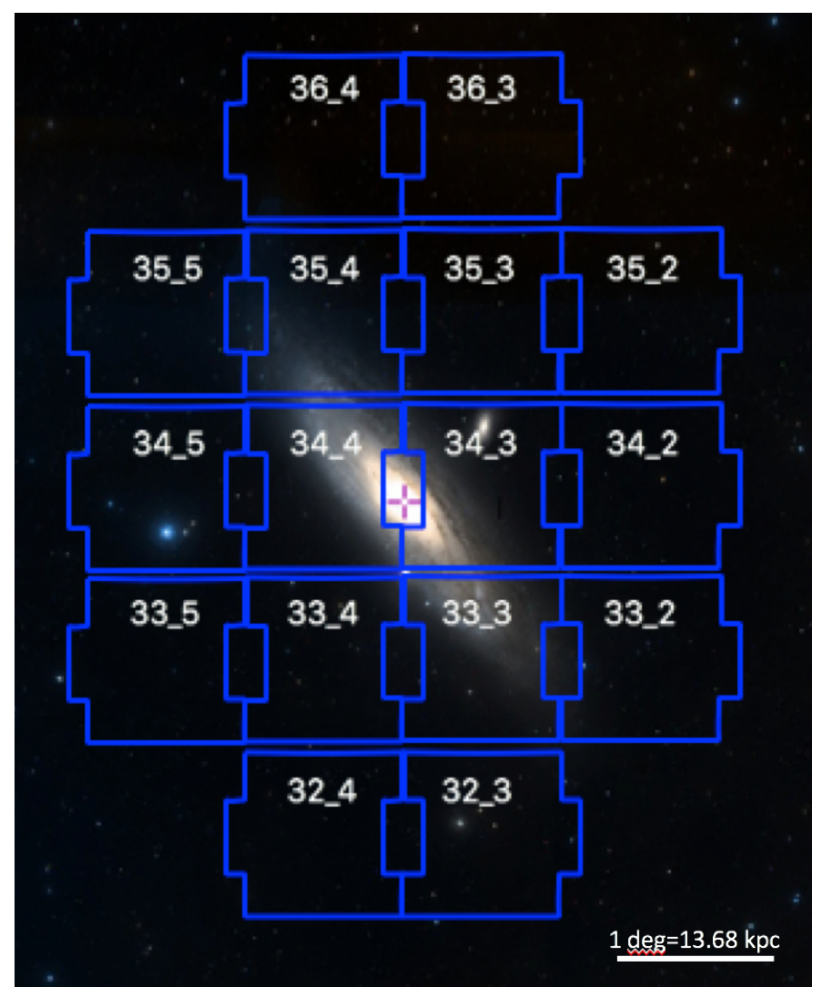

Fig. 1. Fields observed with CFHT MegaCam (in blue, labelled with their observation ID). North is up, east is left. The background image is from SDSS obtained using the Aladin Sky Atlas (Bonnarel et al. 2000).

of $4204 \mathrm{~m}$. MegaCam is comprised of a mosaic of 40 individual $2048 \times 4612$ CCDs, resulting in a contiguous field of view of $0.96 \times 0.94^{\circ}$ with a pixel scale of $0.187^{\prime \prime} /$ pixel. The inner 20-30 kpc radius of the M31 halo were observed through 16 pointings of the MegaCam imager. The fields are shown in Fig. 1. The observations were carried out, under photometric conditions, during two runs: October 9-11 and November 6-7, 2016. Over the course of the observations, seeing varied between $0.5^{\prime \prime}$ and $1.1^{\prime \prime}$, while the airmass varied between 1.03 and 1.48.

M31 is observed through a narrow-band [O III] filter $\left(\lambda_{\mathrm{c}}=\right.$ $5007 \AA, \Delta \lambda=102 \AA$, on-band $)$ and a broad-band $g$-filter $\left(\lambda_{\mathrm{c}}=\right.$ $4750 \AA, \Delta \lambda=1540 \AA$, off-band). The photometry is calibrated with observations of spectrophotometric standard stars. Each onband image generally consists of four dithered exposures with a total exposure time of $1044 \mathrm{~s}$, and each off-band image of three dithered exposures with a total exposure time of $300 \mathrm{~s}$. For some fields there are more dithered images for both on-band and offband, leading to higher exposure times. A summary of the field positions and exposure times for the on-band and off-band exposures is presented in Table 1. The exposure time was chosen such that PNe with an apparent narrow-band magnitude of 6 mag from the bright cut-off $m_{5007}=20.2 \mathrm{mag}$, in the $m_{5007}$ system described by Jacoby (1989), can still be detected.

\subsection{Data reduction}

The data are pre-processed using the Elixir ${ }^{1}$ pipeline (Magnier \& Cuillandre 2004), which accomplishes the bias, flat, and fringe corrections and also determines the photometric zero point of the observations. The zero points for the $[\mathrm{O}$ III] and

\footnotetext{
1 http://www. cfht .hawaii.edu/Instruments/Elixir/home. html
}

Table 1. Summary of the field positions, exposure times, and seeing for the narrow-band (on-band) and broad-band (off-band) images.

\begin{tabular}{ccccccc}
\hline \hline Field & $\begin{array}{c}\alpha(\mathrm{J} 2000) \\
(\mathrm{h}: \mathrm{m}: \mathrm{s})\end{array}$ & $\begin{array}{c}\delta(\mathrm{J} 2000) \\
\left(0:^{\prime}::^{\prime \prime}\right)\end{array}$ & $\begin{array}{c}\operatorname{Exp}_{[\mathrm{O} \mathrm{mI}]}(\mathrm{s}) \\
\operatorname{Exp}_{g} \\
(\mathrm{~s})\end{array}$ & $\begin{array}{c}S_{F W H M} \\
\left({ }^{\prime \prime}\right)\end{array}$ & $\begin{array}{c}m_{5007, \lim } \\
(\mathrm{mag})\end{array}$ \\
\hline 36_4 & $00: 45: 51.9$ & $43: 24: 20.5$ & $4 \times 261$ & $3 \times 100$ & 0.97 & 26.08 \\
36_3 & $00: 40: 14.7$ & $43: 24: 23.7$ & $4 \times 261$ & $3 \times 100$ & 0.95 & 26.26 \\
35_5 & $00: 51: 18.0$ & $42: 21: 48.2$ & $4 \times 261$ & $4 \times 100$ & 0.85 & 26.30 \\
35_4 & $00: 45: 48.0$ & $43: 23: 02.4$ & $8 \times 261$ & $5 \times 100$ & 0.64 & 26.40 \\
35_3 & $00: 40: 17.2$ & $42: 23: 05.6$ & $4 \times 261$ & $6 \times 100$ & 0.66 & 26.17 \\
35_2 & $00: 34: 45.9$ & $42: 21: 57.6$ & $4 \times 261$ & $3 \times 100$ & 0.68 & 26.12 \\
34_5 & $00: 51: 09.5$ & $41: 20: 18.6$ & $4 \times 261$ & $3 \times 100$ & 0.90 & 26.26 \\
34_4 & $00: 45: 44.1$ & $41: 21: 18.7$ & $5 \times 261$ & $3 \times 100$ & 0.85 & 26.16 \\
34_3 & $00: 40: 18.7$ & $41: 21: 21.6$ & $4 \times 261$ & $3 \times 100$ & 0.92 & 25.93 \\
34_2 & $00: 34: 53.3$ & $41: 20: 27.6$ & $6 \times 261$ & $9 \times 100$ & 0.47 & 26.02 \\
33_5 & $00: 51: 02.7$ & $40: 18: 41.6$ & $4 \times 261$ & $3 \times 100$ & 0.77 & 25.94 \\
33_4 & $00: 45: 41.3$ & $40: 19: 48.0$ & $4 \times 261$ & $3 \times 100$ & 0.76 & 25.64 \\
33_3 & $00: 40: 20.9$ & $40: 19: 50.8$ & $4 \times 261$ & $3 \times 100$ & 0.84 & 25.89 \\
33_2 & $00: 35: 00.6$ & $40: 18: 57.6$ & $4 \times 261$ & $3 \times 100$ & 0.54 & 25.90 \\
32_4 & $00: 45: 39.0$ & $39: 18: 16.9$ & $4 \times 261$ & $3 \times 100$ & 0.60 & 26.13 \\
32_3 & $00: 40: 25.1$ & $39: 18: 26.8$ & $4 \times 261$ & $3 \times 100$ & 0.59 & 26.10 \\
\hline
\end{tabular}

Notes. Limiting magnitudes $\left(m_{5007, \text { lim }}\right.$; described in Sect. 3.2) for each field are also provided.

$g$-band frames in $A B$ magnitudes, normalised to a $1 \mathrm{~s}$ exposure, are $Z_{[\text {[Ііा] }}=23.434$ and $Z_{g}=26.5$. For every observed field, a weight map is computed corresponding to each exposure using WeightWatcher ${ }^{2}$ (Marmo \& Bertin 2008). This is used to assign higher weights to pixels that are more reliable compared to their local backgrounds. For each field, the exposures corresponding to the on-band image and those corresponding to the off-band image are then combined using SWARP $^{3}$ (Bertin et al. 2002) to produce the respective on-band and off-band images. The exposures are combined using median-type combination in conjunction with their respective weight maps, while a background subtraction is carried out with a background mesh size of 25 pixels. The images thus obtained are used for source extraction.

\section{Selection of PNe candidates and catalogue extraction}

Having a bright [O III $5007 \AA$ and no continuum emission, extragalactic PNe can be identified as detected objects in the on-band [O III] image that are not detected in the off-band continuum images or that have an excess [O III] $-g$ colour. Additionally, $\mathrm{PNe}$ are typically unresolved at extragalactic distances from ground-based observations and so we only considered point-like objects for analysis. We used the automatic selection procedure developed and validated in Arnaboldi et al. (2002, 2003), which has been optimised for large imaging surveys by Longobardi et al. (2013) and Hartke et al. (2017).

\subsection{Source extraction}

We used SExtractor (Bertin \& Arnouts 1996), a source detection algorithm that detects and measures flux from point-like and extended sources, to detect and carry out photometry of the sources on the images. For each field, we measure the narrowband and broad-band magnitudes, $m_{\mathrm{n}}$ and $m_{\mathrm{b}}$, in dual-image

\footnotetext{
2 http://www.astromatic.net/software/weightwatcher http://www.astromatic.net/software/swarp
} 


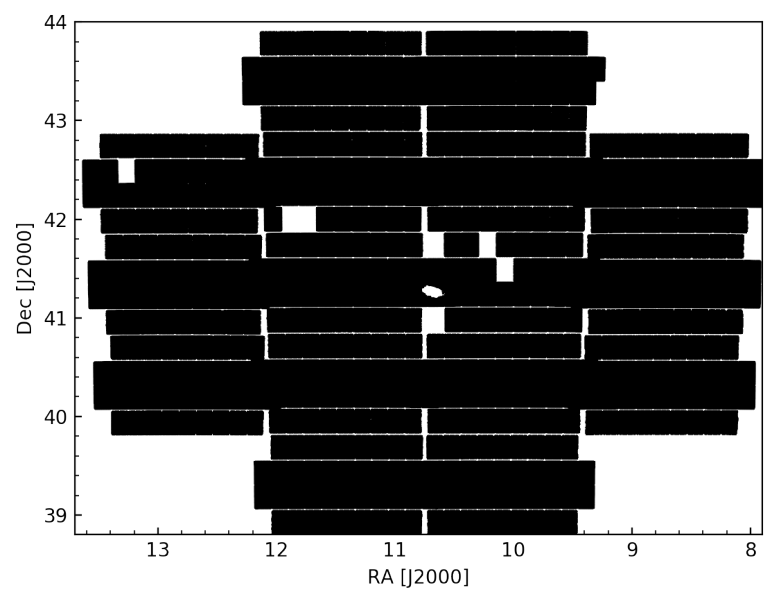

Fig. 2. The effective survey area is shown in black. CCD edges and noisy or saturated regions have been masked.

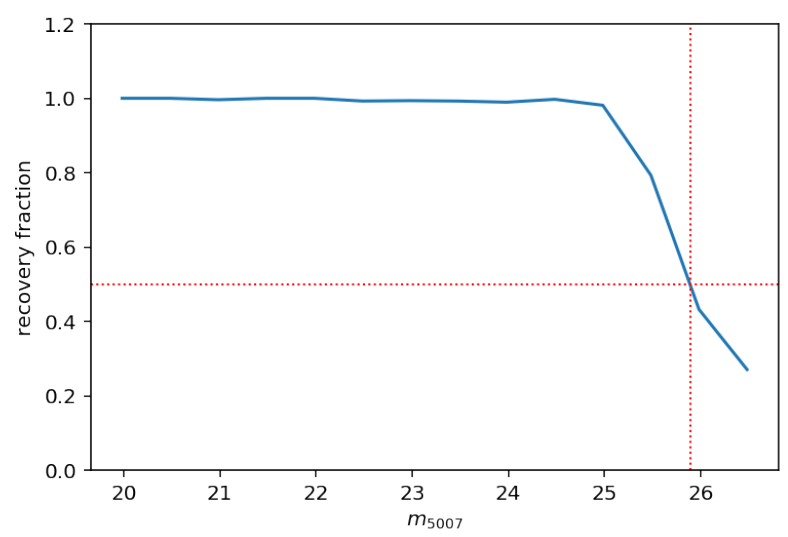

Fig. 3. Recovery fraction of simulated sources for a single field to illustrate the limiting magnitude at the 50\% completeness limit.

mode for sources detected on the narrow-band image. The broadband magnitudes were extracted using the same apertures as the narrow-band image. Sources were detected in the narrowband image requiring that 25 adjacent pixels or more have flux values $1.2 \times \sigma$ rms above the background. Local backgrounds were calculated for the detected sources on apertures having a width of 25 pixels. and magnitudes were measured with different apertures having widths of 15, 17, 19, and 21 pixels. Magnitudes were also measured with an aperture having a width of 5 pixels corresponding to the core of the sources $m_{\text {core }}$. Sources for which a broad-band magnitude could not be detected at the position of the [O III] detection were assigned a $m_{\mathrm{b}}$ corresponding to $1 \times \sigma$ rms above the background in the broad-band image, similar to Arnaboldi et al. (2002). The narrow-band magnitude, $m_{\mathrm{n}}$, in the $\mathrm{AB}$ system was converted to the $m_{5007}$ system as $m_{5007}=m_{\mathrm{n}}+2.27$ (see Appendix A.1 for details). The central part of our survey covering part of the bulge of M31 are mostly saturated. In some observed fields, a few CCDs were noisy. Such noisy regions and CCD edges, affected by dithering and saturation, were masked as detailed in Appendix A.3. The effective survey area is shown in Fig. 2.

\subsection{Limiting magnitude}

In order to determine the limiting magnitude of our sample for each field, we simulated a synthetic point-like population onto the on-band image (with its corresponding exposure time

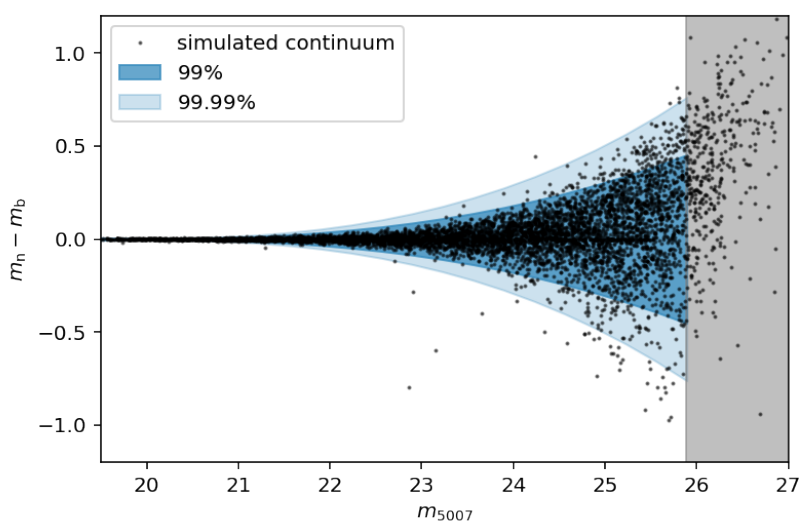

Fig. 4. Colour-magnitude diagram showing the synthetic continuum sources and the $99 \%$ and $99.99 \%$ limits on their positions for a single field. The region beyond the limiting magnitude of this field is shown in grey.

and zero-point) using the Image Reduction and Analysis Facility $\left(\mathrm{IRAF}^{4}\right.$ ) task mkobjects. The synthetic population follows a PNLF as detailed in Ciardullo et al. (1989) and the sources have a Moffat PSF profile as detailed in Appendix A.2. The sources are then extracted. The magnitude aperture most suited to recovering the simulated sources is found to be 15 pixels as detailed in Appendix A.4. The limiting magnitude is defined as the magnitude at which the recovery fraction of the simulated sources drops below $50 \%$ (Fig. 3). This limiting magnitude varies between $m_{5007}=25.64$ for the shallowest observed field, Field\# 33_4, and $m_{5007}=26.4$ for the deepest observed field, Field\# 35_4. The limiting magnitude is provided for each field in Table 1.

\subsection{Colour selection}

We selected PNe candidates based on their position on the $m_{5007}$ versus $m_{\mathrm{n}}-m_{\mathrm{b}}$ colour-magnitude diagram (CMD). These are sources with a colour excess $m_{\mathrm{n}}-m_{\mathrm{b}}<-1$ that are brighter than the limiting magnitude. The colour excess corresponds to an equivalent width $\mathrm{EW}_{\text {obs }}=110 \AA$ (Teplitz et al. 2000) and was chosen in order to limit contamination from background galaxies.

Sources that do not have an excess in $m_{\mathrm{n}}-m_{\mathrm{b}}$ are classified as continuum sources. However, some colour excess may be seen for some of these sources during the source extraction from the images, especially for those with fainter magnitudes. We thus simulated the same synthetic point-like population onto the offband image and checked their position on the extracted $m_{5007}$ versus $m_{\mathrm{n}}-m_{\mathrm{b}}$ CMD (Fig. 4). We calculate the $99 \%$ and $99.99 \%$ limits on their positions in the CMD, below which the probability of detecting continuum sources was reduced to the $1 \%$ and $0.01 \%$ level respectively. Sources with colour excess within the 99.99\% limit are also discarded as possible PNe.

\subsection{Point-like selection}

Planetary nebulae are typically unresolved point-like objects at extragalactic distances, and to differentiate them from extended ones (e.g., background galaxies or other extended objects with strong [O III] emission), we analyse the light distribution of the simulated sources, as described in Sect. 3.2, on the on-band

\footnotetext{
4 http://iraf.noao.edu/
} 

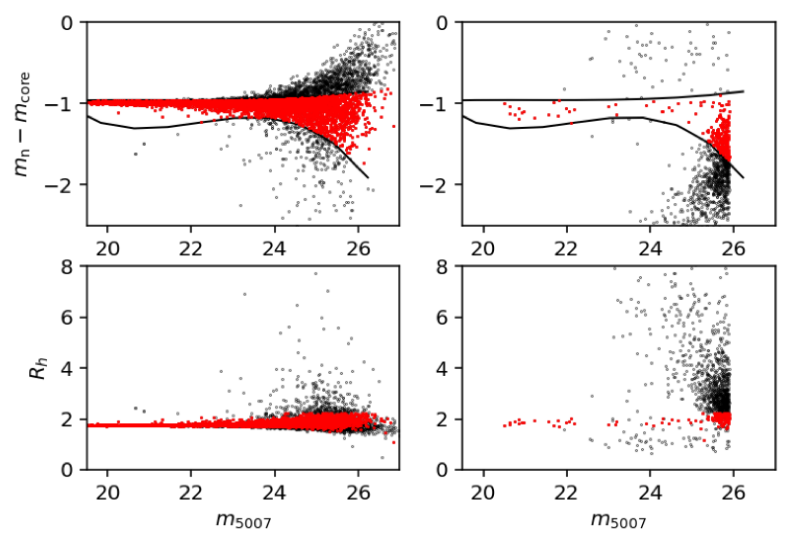

Fig. 5. Top: difference between $m_{\mathrm{n}}$ and $m_{\text {core }}$ for all sources in black and those within the $95 \%$ limits of the simulated population in red for the simulated population (left) and as applied to the real sources (right) for a single field. Bottom: same as the top panel, but for half-light radius, $R_{\mathrm{h}}$.

image. For each field, we use the half-light radius $\left(R_{\mathrm{h}}\right)$ of the simulated sources, which is the radius within which half of the object's total flux is contained, to determine its upper limit $\left(R_{\mathrm{hmax}}\right)$ corresponding to $95 \%$ of the simulated population. We considered sources as point-like if they satisfied the following two criteria: (i) they have a half-light radius such that $1<R_{\mathrm{h}}<R_{\mathrm{hmax}}$, and (ii) they fall in the region where the difference between $m_{\mathrm{n}}$ and $m_{\text {core }}$, magnitude of the source for flux within an aperture of 5 pixels, is within the $95 \%$ limit of the simulated population. The point-like selection criteria are shown in Fig. 5, as applied to the colour-selected simulated population and to the real sources.

In order to estimate the number of continuum sources that may be misidentified as PNe in any field, we count the number of point-like continuum sources (excluded as PNe by the colour selection criteria) and multiply by $0.01 \%$. We estimate that in each field, our identified PNe sample may be contaminated by $2-4$ continuum sources that lie in the faint magnitudes $>25$.

\subsection{PNe catalogue}

For each field, the [O III] sources that are brighter than the limiting magnitude and fulfil both the point-like and colour selection criteria are considered as PNe candidates. They are shown in the CMD in Fig. 6 for a single field. Since the bandwidth of the broad-band filter also covers that of the narrow-band filter, the bright PNe are expected to show some remnant flux in the broad band as well. This remnant broad-band flux is in the ratio of the filter widths and leads to nearly a constant colour excess, $m_{\mathrm{n}}-m_{\mathrm{b}}=2.5 \log \left(\frac{\Delta \lambda_{[\mathrm{Om}]}}{\Delta \lambda_{g}}\right)=-2.95$, for the bright PNe. The observed constant colour excess is slightly less negative due to the flux contributed to the broad band from the [O III] $4959 \AA$ line which is expected to be $\sim 1 / 3$ of the brightness of the [O III] $5007 \AA$ line in PNe, as seen in Fig. 6. The final catalogue of confirmed $\mathrm{PNe}$ is then checked for spurious sources by eye. Regions of spurious sources, typically caused by saturated stars, are masked and the final catalogue of confirmed $\mathrm{PNe}$ is obtained for each field. Counting the PNe identified in the overlapping regions of adjacent fields only once, we identify an unprecedented 4289 PNe in M 31 in our survey. Their spatial distribution, overlaid on a map of RGB stars identified by the PAndAS survey, is shown in Fig. 7. Our survey is uniformly

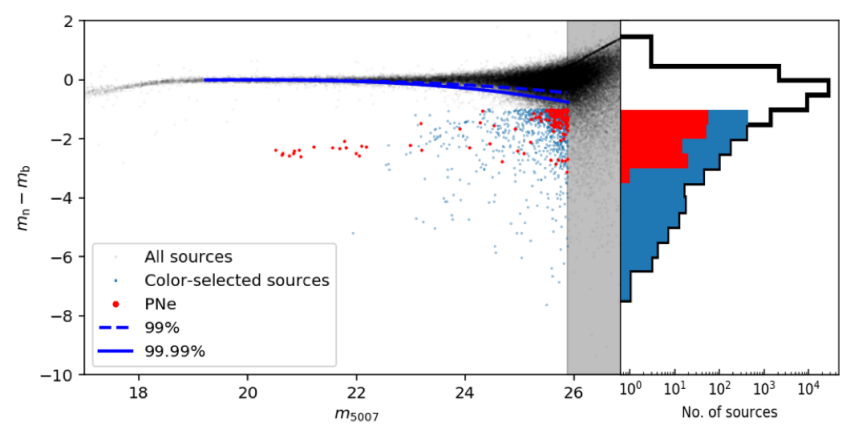

Fig. 6. Colour-magnitude diagram for a single field showing all the detected sources (brighter than the limiting magnitude) in black, the colour-selected sources in blue, and identified PNe in red. The $99 \%$ and 99.99\% limits for the continuum sources are shown in blue, while the region beyond the $50 \%$ completeness limit is shown in grey. The histogram (in logarithmic scale) shows clearly the number of PNe recovered as a function of colour with all the detected sources (brighter than the limiting magnitude) in black, the colour-selected sources in blue, and identified $\mathrm{PNe}$ in red.

complete (in the effective survey area) down to $m_{5007}=25.64$ which is the $50 \%$ completeness limit of the shallowest field (Field\# 33_4). However photometric depth varies with fields and we find PNe down to $m_{5007}=26.4$ for the deepest field (Field\# 35_4).

\subsection{Completeness correction}

Our colour and point-like selection criteria would exclude a number of $\mathrm{PNe}$ that are affected by photometric errors, especially in the fields covering the bright M31 disc. Thus, in order to determine the selection completeness of our extracted sample, we follow the procedure outlined in Longobardi et al. (2013) and Hartke et al. (2017). We simulated a population of $10^{4}$ point-like sources (Sect. 3.2) on the narrow-band image. On the broad-band image, we also simulate sources at the same positions with their fluxes scaled down by the ratio of the filter widths ${ }^{5}$. We then use SExtractor in dual-mode to simultaneously extract $m_{\mathrm{n}}$ and $m_{\mathrm{b}}$ of these simulated sources. Figure 8 shows the CMD of the simulated population for a single field. The colour excess remains nearly constant for the bright simulated sources, as expected from the ratio of their filter widths, but many are missed at fainter magnitudes. We can thus determine the selection completeness of the simulated population at different magnitude ranges for each field. To a single candidate extracted in a given field, we assign the value of the selection completeness at that magnitude as a probability of being detected after the selection effects. We also compute the detection completeness of the simulated population at different magnitude ranges from the recovery fraction and similarly assign a detection probability to each PN candidate extracted in each field. The completeness correction is thus obtained from both the selection and the detection probability (see further details in Sect. A.5).

\subsection{Comparison with the Merrett et al. (2006) PNe catalogue}

We identify those sources in our PNe catalogue that have a counterpart in the catalogue of PNe identified by M06 by matching them spatially within a $3^{\prime \prime}$ aperture (Fig. 9). The astrometry of

\footnotetext{
5 We neglect the contribution from the [O III] $4959 \AA$ line in the broad-
} band image. 


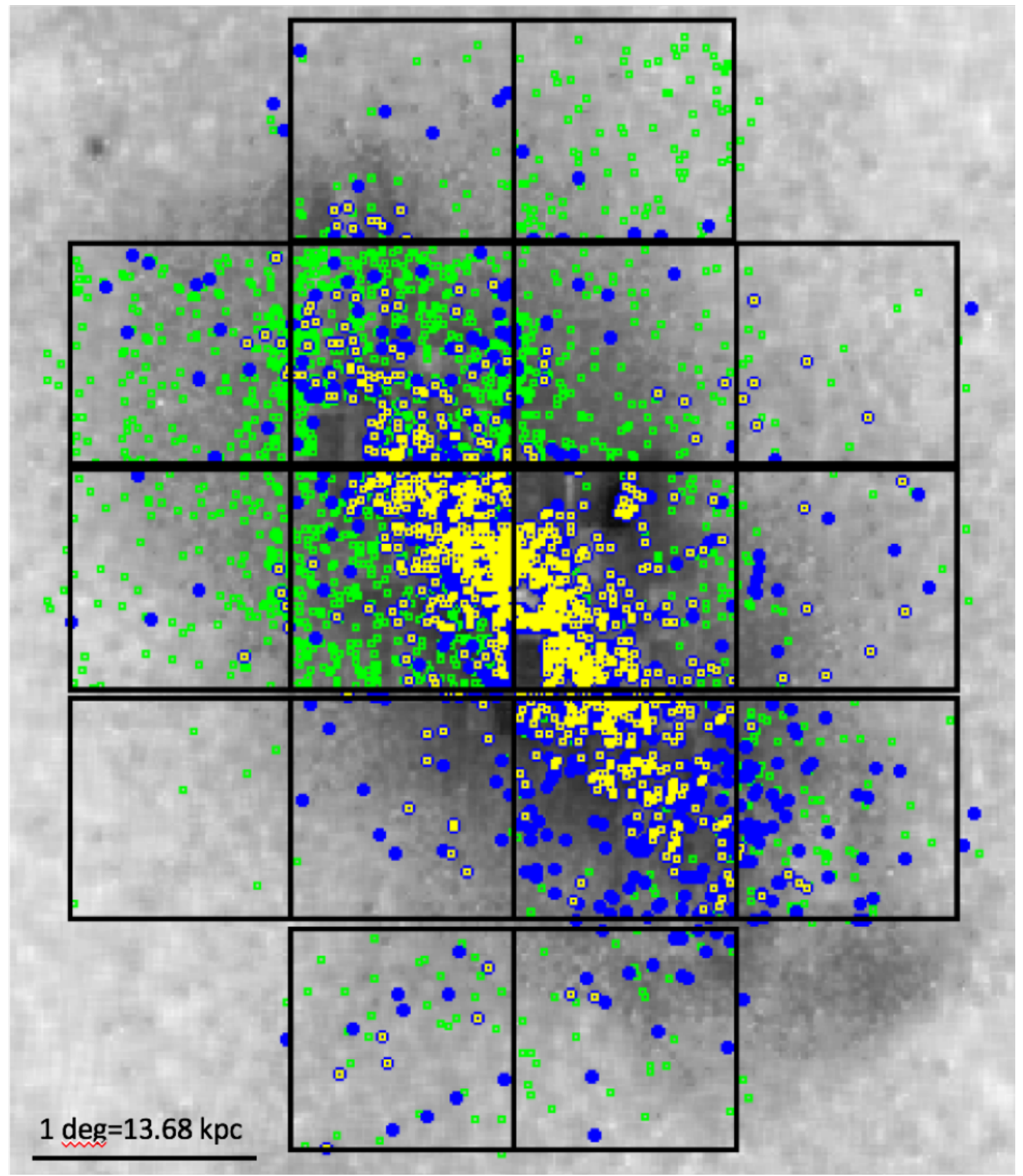

Fig. 7. Planetary nebulae identifed by the survey are overlaid on the map of RGB stars from the PAndAS survey. PNe brighter than $m_{5007}=$ $25.64 \mathrm{mag}$, which is the $50 \%$ completeness limit of the shallowest field (Field\# 33_4), are shown in blue. PNe fainter than $m_{5007}=25.64$ mag, with photometric depth varying with field, are shown in green. The M06 PNe, re-identified by our survey, are shown in yellow. North is up, east is left.

the M06 sample is reliable up to 3" (Veyette et al. 2014). We match 1099 such sources (in yellow in Fig. 7). The narrow-band magnitudes of the matched $\mathrm{PNe}$ in our survey are $0.045 \mathrm{mag}$ brighter than the corresponding value in M06, well within the photometric uncertainty of the M06 sample. We thus validate the [O III] $5007 \AA$ photometry of our PNe with the sample of M06. The photometry of the M06 PN sample is not very accurate for fainter sources because the PN.S instrument used by M06 has not been optimised for photometry measurements, but rather for the measurement of radial velocities.
While we find most of the PNe found by M06 in the observed fields, we miss quite a few in the regions that we masked, mainly in the crowded bulge and the CCD edges. In our survey area, we recover $82.22 \%$ of the M06 PNe. Up to $25 \%$ of the M06 PNe candidates were actually $\mathrm{H}$ II regions in the M31 disc (Veyette et al. 2014). The spatial resolution of MegaCam bolstered by the favourable seeing allows us to improve the image quality and obtain an accurate PSF to identify $\mathrm{PNe}$ in M31. The $\mathrm{H}_{\text {II }}$ regions would appear as extended objects and discarded as $\mathrm{PNe}$ by our point-like selection. 


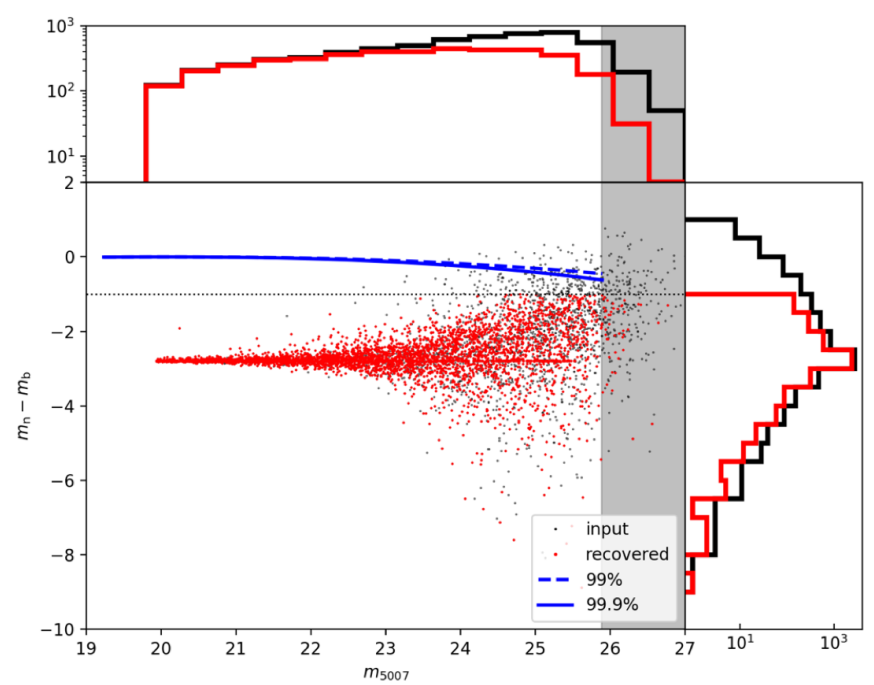

Fig. 8. Colour-magnitude diagram for a single field showing all the simulated sources in black and those recovered as PNe in red (see Sect. 3.6). The $99 \%$ and $99.99 \%$ limits for the continuum sources are shown in blue, while the region beyond the limiting magnitude is shown in grey. The dotted black line shows the colour selection adopted to limit contamination from background galaxies. The histograms (in logarithmic scale) show the number of PNe recovered as a function of colour (right) and magnitude (top) with the simulated sources in black and those recovered as PNe in red.

We investigate the contamination of our PNe catalogue by $\mathrm{H}_{\text {II }}$ regions in the next section and further discuss it in Sect. 7.1. Thus, the $82.22 \%$ recovery fraction of the M06 PNe is driven by the tighter morphological constraints on the light distribution of the detected [O III] sources in our survey. Since the limiting magnitude of our survey is 1.5-2 mag fainter than that of M06, we find a much larger number of new PN candidates in the overlapping area.

\section{Counterparts in HST imaging}

To test the image quality of our PNe survey, and to estimate the possible contamination from $\mathrm{H}_{\text {II }}$ regions and other sources, we identified PNe counterparts in the HST data available from the Panchromatic Hubble Andromeda Treasury (PHAT; Dalcanton et al. 2012) covering a portion of the M 31 disc.

\subsection{The Panchromatic Hubble Andromeda Treasury}

The PHAT $^{6}$ survey covers $\sim 1 / 3$ of the star-forming disc of M 31 in six bands from the near-UV to the near-IR using the HST imaging cameras (WFC3/IR, WFC3/UVIS, and ACS/WFC cameras). It combines the wide-field coverage typical of groundbased surveys with the precision of HST observations. The overall survey strategy, initial photometry, and data quality (DQ) assessments are described in detail in Dalcanton et al. (2012). We utilise the second generation of photometric measurements of the resolved stars in the PHAT imaging (Williams et al. 2014), which takes advantage of all available information by carrying out photometry simultaneously in all six filters, resulting in a significant increase in the depth and accuracy the photometry over that presented in Dalcanton et al. (2012).

\footnotetext{
6 https://archive.stsci.edu/prepds/phat
}

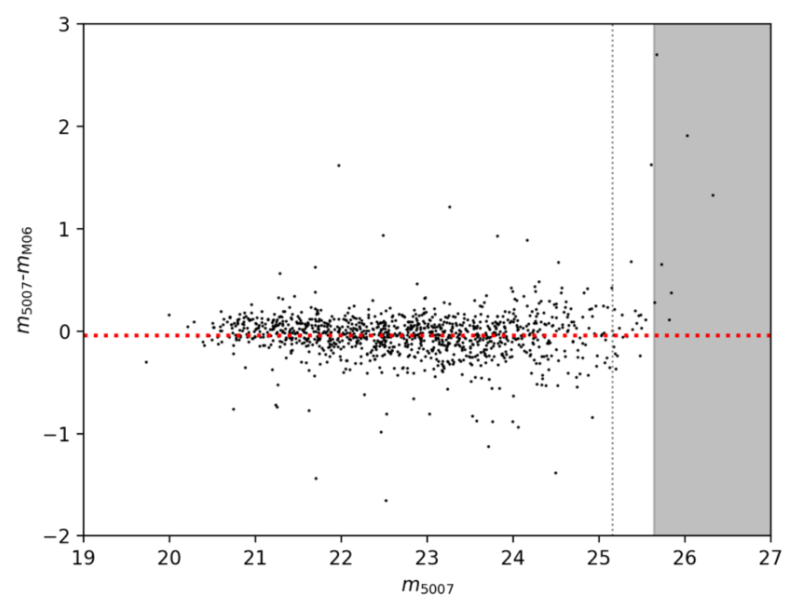

Fig. 9. Difference between the narrow-band magnitudes of this work and M06 obtained for the matched sources, plotted against $m_{5007}$. The red dashed line corresponds to the mean offset. The region beyond the limiting magnitude of the shallowest field (Field\# 33_4) is shown in grey. The grey dotted line shows the $90 \%$ completeness limit of the shallowest field.

\subsection{Finding PNe counterparts in PHAT}

Veyette et al. (2014) conducted a search for M06 PNe counterparts in 16 of the 23 bricks in the PHAT footprint. They find that $\sim 25 \%$ of the PNe identified by M06 in the M31 disc are either $\mathrm{H}$ II regions resolved in HST or stellar contaminants. They utilised the photometry presented in Dalcanton et al. (2012). They found a linear relation between the M06 $m_{5007}$ magnitude and the PHAT F475W magnitude given by

$\mathrm{F} 475 \mathrm{~W}=-0.2240+1.0187 \times m_{5007}$.

We adopt a similar method for identifying PHAT counterparts to our PNe in the entire PHAT footprint. However, we search for our counterparts in the updated PHAT photometry from Williams et al. (2014). We only consider those sources from the v2 star files whose square of the sharpness parameter in the F475W filter is below 0.2, to avoid cosmic rays and extended objects. The selection is made on the basis of the following parameters: 1 . difference between F475W mag and the F475W mag expected from the $m_{5007}$ using the relation described by Veyette et al. (2014); 2. the F475W-F814W colour; 3. the positional separation between the PNe and the PHAT counterpart; and 4. the roundness of the PSF. To automatically select candidates on the basis of these parameters, we construct an initial training set with the PNe in common with Veyette et al. (2014). We calculated the average value $\left(\bar{X}_{\mathrm{PN}}\right)$ and $1 \sigma$ spread $\left(\sigma_{\mathrm{PN}}\right)$ of each identification parameter. We found that the separation is within $0.7^{\prime \prime}$. For every PNe, the differences between the training set $\left(\bar{X}_{\mathrm{PN}}\right)$ and the source $\left(X_{\mathrm{S}}\right)$ parameter values were normalised by the $\sigma_{\mathrm{PN}}$ of the parameter values for each source in PHAT within 1" of our PNe location. These normalised parameters are of the form

$Z_{\mathrm{x}}=\frac{\left|X_{\mathrm{s}}-\bar{X}_{\mathrm{PN}}\right|}{\sigma_{\mathrm{PN}}}$

We obtain the sum of these normalised parameters, with half the weight given to roundness, as the merit function $Z_{\mathrm{PN}}$ :

$Z_{\mathrm{PN}}=\frac{Z_{\mathrm{F} 475 \mathrm{~W}}+Z_{\text {colour }}+Z_{\mathrm{sep}}+0.5 \times Z_{\text {round }}}{3.5}$. 


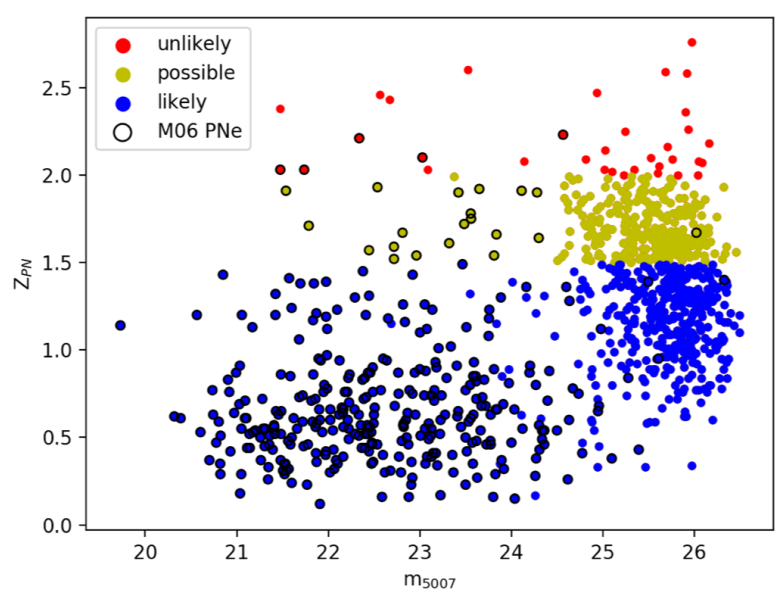

Fig. 10. Merit function, $Z_{\mathrm{PN}}$, of the PHAT-matched PNe plotted against $m_{5007}$. The "likely", "possible", and "unlikely" PNe are shown in blue, yellow, and red, respectively. The PNe previously found by M06 are ringed in black.

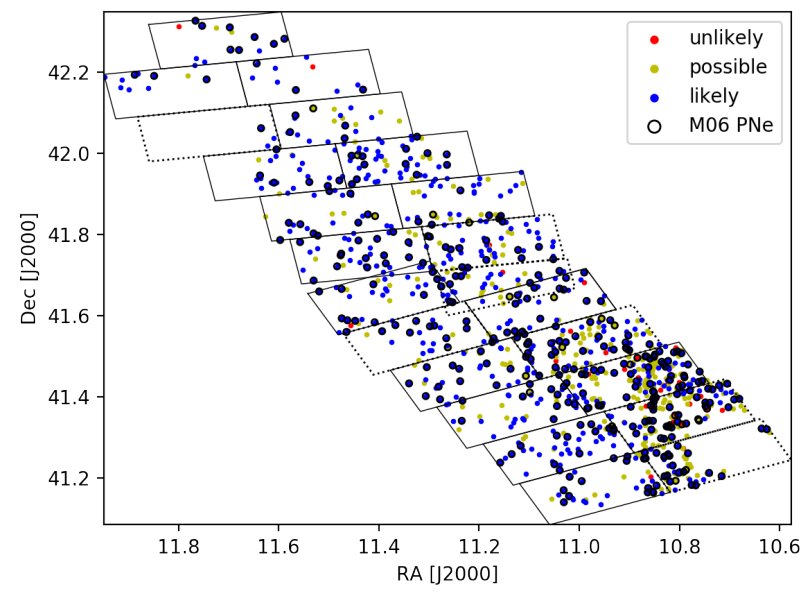

Fig. 11. Spatial distribution of the PHAT-matched $\mathrm{PNe}$ in the PHAT footprint. The solid bricks are those previously analysed by Veyette et al. (2014), while the dashed ones have been analysed here for the first time. The "likely", "possible", and "unlikely" PNe are shown in blue, yellow, and red, respectively. The PNe previously found by M06 are ringed in black.

For each PNe, the PHAT source with the lowest value of $Z_{\mathrm{PN}}$ is considered as the counterpart. The distribution of the assigned $Z_{\mathrm{PN}}$ with the $m_{5007}$ of the PHAT-matched PNe is shown in Fig. 10. Thresholds in $Z_{\mathrm{PN}}$ are used to classify the PNe based on the $Z_{\mathrm{PN}}$ assigned to those PHAT sources which were not PNe counterparts. PNe with $Z_{\mathrm{PN}}<1.5$ are classified as "likely", those with $1.5<Z_{\mathrm{PN}}<2$ are classified as "possible" and those with $Z_{\text {PN }}>2$ are classified as "unlikely". The likely PHAT sources have a significantly lower $Z_{\mathrm{PN}}$ than that of other PHAT sources in the search region, but those that are possible do not stand out quite so much. Those classified as unlikely may not be $\mathrm{PNe}$ at all as stellar PHAT sources can have $Z_{\mathrm{PN}} \sim 2$, even though most of them have $Z_{\mathrm{PN}}>4$. The spatial distribution of PHAT PNe counterparts is presented in Fig. 11.

\subsection{Characteristics of the PHAT-matched PNe}

Of the 1023 PNe in the PHAT footprint, 700 are classified as likely, 292 as possible and 31 as unlikely. True colour images of three PNe, one for each classification, is shown in Fig. 12. None of the PHAT PNe are resolved as $\mathrm{H}$ II regions. The 31 unlikely PNe ( $\sim 3 \%$ of the PHAT PNe) may be stellar contaminants. Of the $\mathrm{H}$ II regions spectroscopically identified by Sanders et al. (2012), 81 are present in the PHAT footprint. Many of these were misidentified by M06 as PNe. We misidentify only three of these $\mathrm{H}_{\text {II }}$ regions (probably compact $\mathrm{H}_{\mathrm{II}}$ regions) as $\mathrm{PNe}$, which is a testament to the photometric quality and improved spatial resolution of our survey.

The mean positional separation of our PNe and their PHAT counterparts is $0.27^{\prime \prime}$ which is a testament to the accurate astrometry of the image and the Elixir pipeline greatly benefiting our survey. Figure 13 shows the variation in separation with $m_{5007}$. The separation remains largely uniform throughout the range of $m_{5007}$. The correlation between $m_{5007}$ and the PHAT F475W mag is also seen for our PNe (Fig. 14), although there is larger dispersion at the faint end. Similarly the variation in the PHAT F475W mag with the F475W-F814W colour (Fig. 15) shows that most of the faint sources that are classified as possible and unlikely have a higher F475W-F814W colour. The dispersion in F475W mag and the higher colour for some of the faint sources may be due to the [O III] $5007 \AA$ line not being as prominent as the continuum flux in F475W for the fainter PNe.

\section{Planetary nebula luminosity-specific frequency}

The PNe population follows the sampled (bolometric) luminosity of its parent stellar population. The PN luminosity-specific frequency ( $\alpha$-parameter; Jacoby 1980) is the ratio of the total number of PNe, $N_{\mathrm{PN}}$, to the total bolometric luminosity of the parent stellar population, $L_{\mathrm{bol}}$, given by

$\alpha=\frac{N_{\mathrm{PN}}}{L_{\mathrm{bol}}}=B \tau_{\mathrm{PN}}$,

where $B$ is the specific evolutionary flux ( $\operatorname{stars~} \mathrm{yr}^{-1} L_{\odot}^{-1}$ ) and $\tau_{\mathrm{PN}}$ is the PN visibility lifetime (Buzzoni et al. 2006). The $\alpha$-parameter value provides the number of PNe expected per unit bolometric light. We determine the radial PN number density profile and by comparison with the stellar surface brightness profile of M31, derived from broad-band photometric studies, we compute the $\alpha$-parameter value, similarly to the procedure followed for M 87 (Longobardi et al. 2013) and M 49 (Hartke et al. 2017).

\subsection{Radial PN number density profile}

Figure 16 shows the distribution of $\mathrm{PNe}$ on the sky together with 15 elliptical bins aligned using the known position angle, $\mathrm{PA}=38^{\circ}$ of $\mathrm{M} 31$ and its ellipticity $=0.73$ (Walterbos \& Kennicutt 1988). The binning was chosen such that each bin contains the same number of PNe brighter than $m_{5007}=25.64$, which is the $50 \%$ completeness limit of the shallowest field (Field\# 33_4). These bins correspond to $0.65-26.13 \mathrm{kpc}$ radial distances projected on the minor axis of M31, $R_{\min }$. The PN logarithmic number density profile is defined as

$\mu_{\mathrm{PN}}(r)=-2.5 \log _{10} \Sigma_{\mathrm{PN}}(r)+\mu_{0}$,

where $\mu_{0}$ is a constant in order to match the PN number density profile with the stellar surface brightness profile, and $\Sigma_{\mathrm{PN}}$ is the completeness-corrected PN number density which is in turn given by

$\Sigma_{\mathrm{PN}}(r)=\frac{N_{\mathrm{PN}, \mathrm{corr}}(r)}{A(r)}$ 

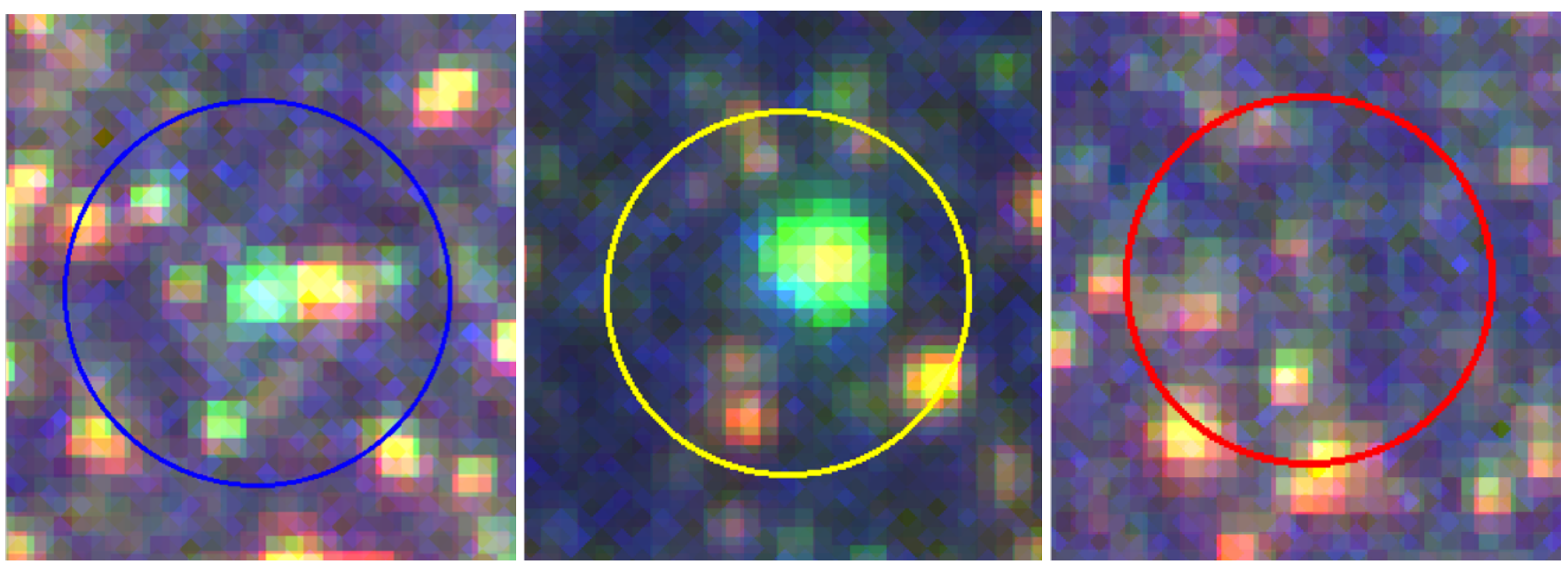

Fig. 12. True colour images of PHAT-matched PNe. F814W, F475W, and F336W were used for the red, green, and blue images, respectively. One "likely" (left), "possible" (centre), and "unlikely" (right) PN is shown. The circle denotes 0.5" around the PN position.

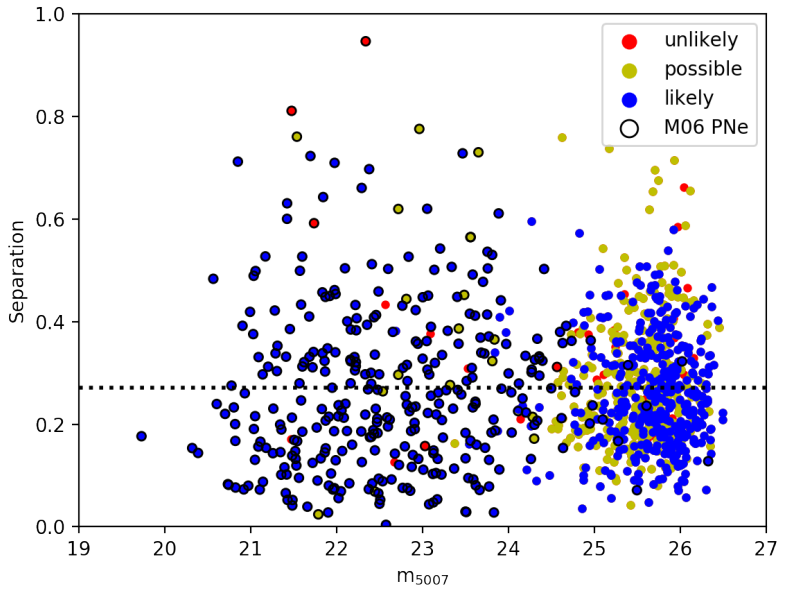

Fig. 13. Positional separation between the PNe and their PHAT counterparts plotted against $m_{5007}$. The dashed line shows the mean positional separation. The "likely", "possible", and "unlikely" PNe are shown in blue, yellow, and red respectively. The PNe previously found by M06 are ringed in black.

and $N_{\mathrm{PN}, \text { corr }}$ is the completeness-corrected PN number in any elliptical bin. The completeness correction accounts for both detection and colour incompleteness, detailed in Sect A.5. The parameter $A(r)$ is the observed area in any elliptical bin. We obtain the density profile using only the PNe within 2.5 mag of the bright cut-off $\left(m_{5007}<22.56\right)$. The resulting PN density profile in each elliptical bin is matched to the $V$-band surface brightness profile, obtained by Irwin et al. (2005) using photometry and number counts along the minor axis of M31. It is shown in Fig. 17. We compute $\mu_{0}=11.085 \pm 0.004$. Courteau et al. (2011) decomposed the M31 luminosity profile into the bulge, disc, and halo components finding that the halo component becomes significant from $R_{\min } \sim 8 \mathrm{kpc}$ and it dominates the luminosity profile over the disc from $R_{\min } \sim 11 \mathrm{kpc}$. The PN density profile follows the surface brightness profile from $R_{\min } \sim$ $2-8 \mathrm{kpc}$, where the disc dominates the luminosity profile. The M $31 B-V$ colour, available out to $R_{\min } \sim 10 \mathrm{kpc}$, shows a gradient towards bluer colours at larger radii (Walterbos \& Kennicutt 1988; Tempel et al. 2010). We find a flattening of $\mu_{\mathrm{PN}}$ with respect to the observed surface brightness profile at larger radial distances. This correlates with the $B-V$ colour gradient and the

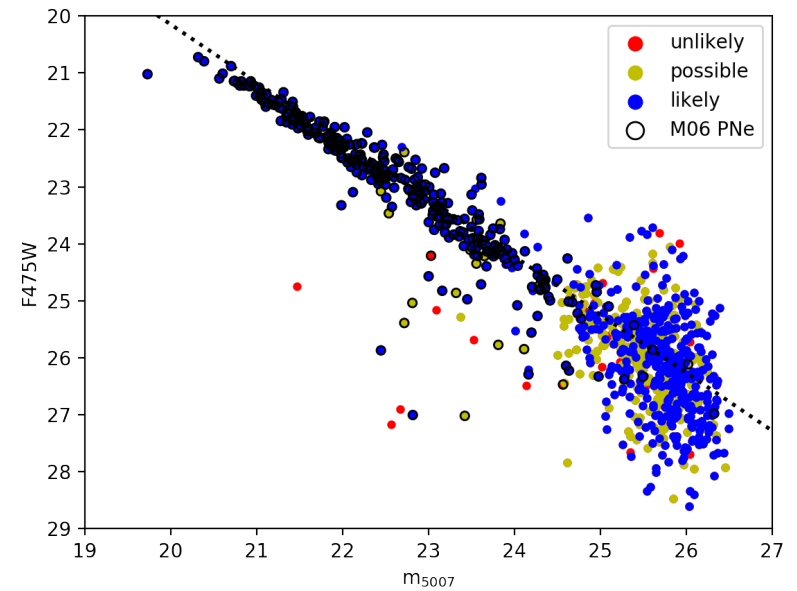

Fig. 14. F475W mag of the PHAT-matched PNe plotted against $m_{5007}$. The dashed line shows their relation described by Veyette et al. (2014). The "likely", "possible", and "unlikely" $\mathrm{PNe}$ are shown in blue, yellow, and red respectively. The PNe previously found by M06 are ringed in black.

increasing dominance of the halo component in the light-profile. Additionally, the light from the inner-halo substructures is underestimated by the surface brightness profile obtained along the minor axis of M31 at larger radii. This may also lead to a flattening of $\mu_{\mathrm{PN}}$.

\subsection{PN luminosity-specific number: the $\alpha$-parameter}

We can compute the value of $\alpha$ for the observed M31 PNe from $\mu_{0}$ using the relation

$\alpha=\frac{1}{s^{2}} 10^{0.4\left(\mu_{0}-K-\left(\mathrm{BC}_{\odot}-\mathrm{BC}_{V}\right)\right)}$,

where the $K=26.4 \mathrm{mag} \operatorname{arcsec}^{-2}$ is the $V$-band conversion factor to physical units $L_{\odot} \mathrm{pc}^{-2}, \mathrm{BC}_{\odot}=-0.07$ is the solar bolometric correction, and $s=D / 206265$ is a scale factor related to the galaxy distance $D$. A fixed value of $\mathrm{BC}_{V}=0.85$ can be assumed with $10 \%$ accuracy based on the study of stellar population models for different galaxy types (irregular to elliptical; see Buzzoni et al. 2006).

For the PNe within 2.5 mag of the bright cut-off, we obtain $\alpha_{2.5}=(40.55 \pm 3.74) \times 10^{-9} \mathrm{PN} L_{\odot, \text { bol }}^{-1}$. For a PN population 


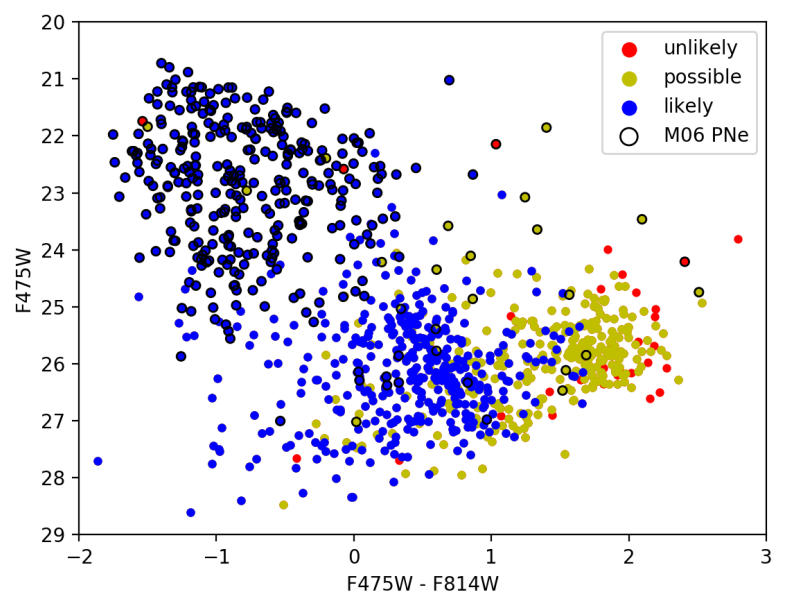

Fig. 15. F475W mag of the PHAT-matched PNe plotted against its F475W-F814W colour. The "likely", "possible", and "unlikely" PNe are shown in blue, yellow, and red respectively. The PNe previously found by M06 are ringed in black.

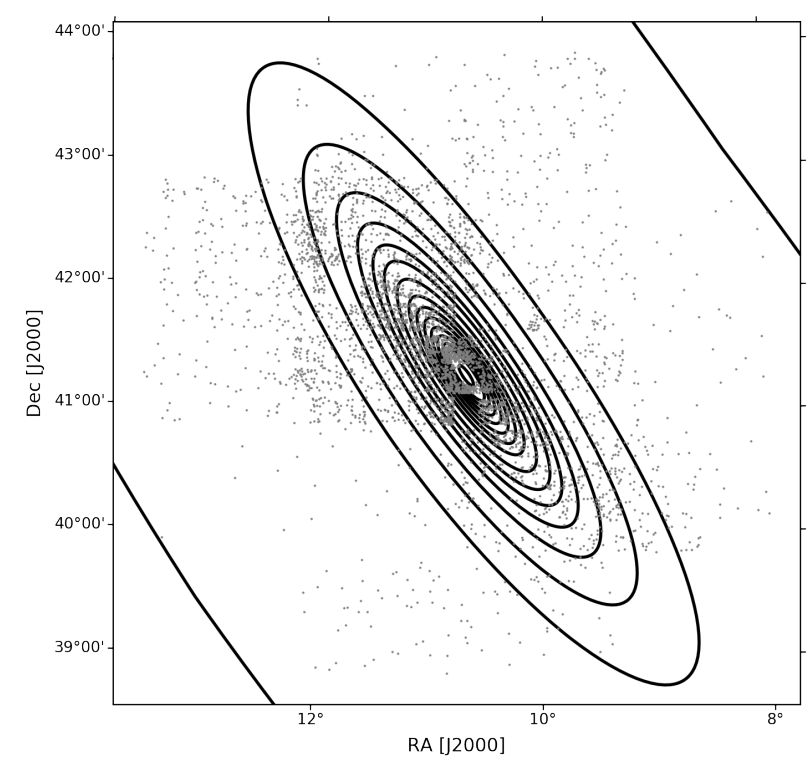

Fig. 16. Planetary nebulae are shown spatially divided into elliptical bins containing an equal number of PNe brighter than the 50\% completeness limit of the shallowest field (Field\# 33_4).

following the PNLF described by Ciardullo et al. (1989), this corresponds to a $\log \alpha=\log \left(\alpha_{2.5} / 0.1\right)=-6.39 \pm 0.04$ and $\tau_{\mathrm{PN}}=(22527.78 \pm 207.78) \mathrm{yr}$ (based on relations described in Buzzoni et al. 2006). M06 obtained $\alpha_{2.5}=(15 \pm 2) \times 10^{-9} \mathrm{PN}$ $L_{\odot, \text { bol }}^{-1}$, but only within $1.8 \mathrm{kpc}$ of the minor-axis radius. Scaling our PN number density profile to the surface brightness profile only within this interval, we find $\mu_{0}=10.33 \pm 0.01$ corresponding to $\alpha_{2.5}=(20.16 \pm 1.87) \times 10^{-9} \mathrm{PN} L_{\odot, \text { bol }}^{-1}$, which is closer to the value obtained by M06. This may be due to the bulge, disc, and halo components of M 31 having differing $\alpha$-parameter values.

\subsection{Three-component photometric model for $M 31$}

Longobardi et al. (2013) and Hartke et al. (2017) also saw differences in the $\alpha$-parameter values associated with different components of the surface brightness profile, although they looked at halo and intra-group light components. As in their procedure, we describe a photometric model for the predicted PN surface

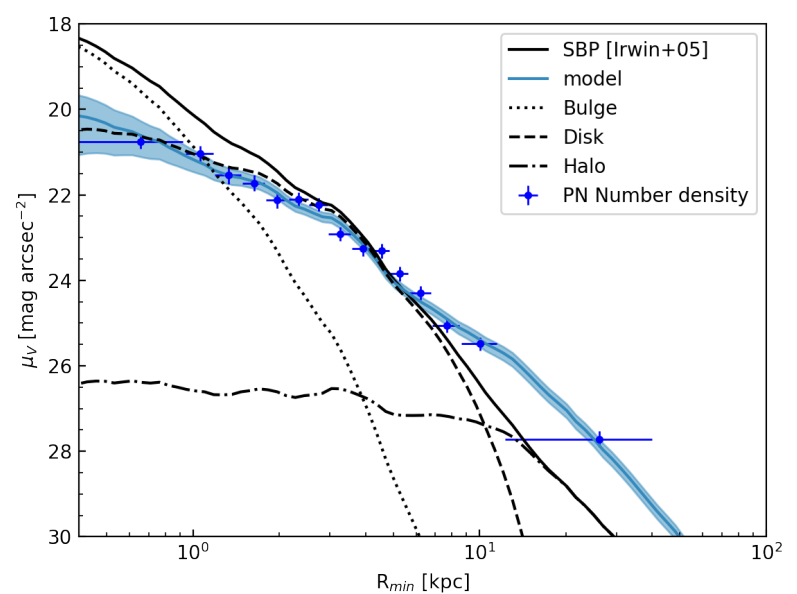

Fig. 17. Radial surface brightness profile of M31 (Irwin et al. 2005) (in black) and the PN density using only the PNe within 2.5 mag of the bright cut-off, obtained at different elliptical bins (in blue). The Courteau et al. (2011) decomposition of the M31 luminosity profile into the bulge, and disc and halo components scaled to the $V$-band surface brightness profile are also shown. The three-component photometric model for the predicted PN surface density is shown in blue.

density as

$\widetilde{\Sigma}_{\mathrm{PN}}(r)=\left(\alpha_{2.5, \text { bulge }} I_{\text {bulge }}(r)+\alpha_{2.5, \text { disk }} I_{\text {disk }}(r)+\alpha_{2.5 \text {,halo }} I_{\text {halo }}(r)\right) s^{2}$,

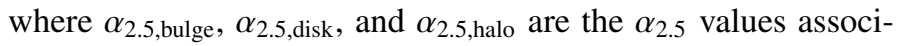
ated with the bulge, disc, and halo components of M31, respectively; $I_{\text {bulge }}, I_{\text {disk }}$, and $I_{\text {halo }}$ are the surface brightness profiles of the bulge, disc, and halo components of M 31, respectively, as per the Courteau et al. (2011) decomposition of the M 31 luminosity profile into the bulge, disc, and halo components, scaled to the $V$ band surface brightness profile observations by Irwin et al. (2005).

We simultaneously fit the different $\alpha$-parameter values of the photometric model to the observed PN surface density to obtain $\alpha_{2.5, \text { bulge }}=(5.28 \pm 6.25) \times 10^{-9} \mathrm{PN} L_{\odot, \text { bol }}^{-1}, \alpha_{2.5 \text {, disk }}=(39.16 \pm$ $3.33) \times 10^{-9} \mathrm{PN} L_{\odot, \text { bol }}^{-1}$ and $\alpha_{2.5 \text {, halo }}=(273.89 \pm 41.31) \times 10^{-9} \mathrm{PN}$ $L_{\odot \text {,bol }}^{-1}$. The model is also shown in Fig. 17. While the $\alpha_{2.5 \text {,bulge }}$ is not very well constrained, we find the $\alpha_{2.5 \text {,disk }}$ is quite close to the $\alpha_{2.5}$ obtained for the whole PN sample, which is expected since the surface brightness profile of M 31 is dominated by the disc component in our survey. We also find that $\alpha_{2.5 \text {, halo }} \sim 7 \alpha_{2.5 \text {, disk }}$. The observed variation in $\alpha$-parameter values in different galaxies that spans almost two orders of magnitude was studied by Buzzoni et al. (2006), who show that late-type spiral and irregular galaxies with bluer $B-V$ colours are expected to have larger $\alpha$-parameter values than the redder early-type galaxies. Their analysis is based on population synthesis models of galaxies with different ages, metallicities, and morphological types. The different stellar populations in these galaxies thus exhibit very different $\alpha$-parameter values. Hartke et al. (2017) also found that $\alpha$-parameter values for the intra-group light of M 49 is three times larger than that of its halo. Hartke et al. (2018) later confirmed that the halo PNe and intra-group light PNe were kinematically different, and correspond to two separate parent stellar populations. We can thus infer that the larger $\alpha$-parameter value of the bluer halo of M 31 may indicate that the stellar population of the inner halo is different from that of the disc. While the bluer halo is thus expected to have a higher $\alpha_{2.5}$ than the disc, its absolute value measured for the M 31 halo is the highest observed in any galaxy. 


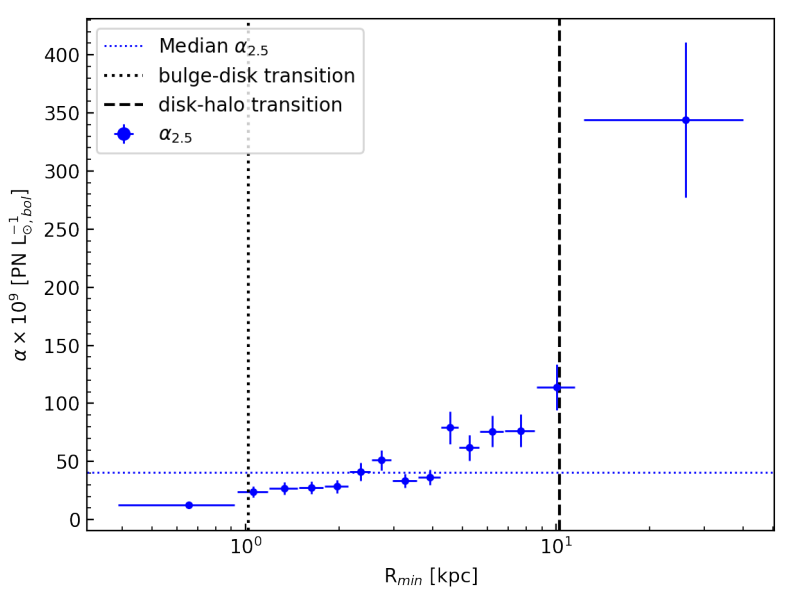

Fig. 18. Values of $\alpha_{2.5}$ obtained at different elliptical bins (in blue). The dotted black line and the dashed black line show the bulge-disc transition and the disc-halo transition, respectively, from the Courteau et al. (2011) decomposition of the M31 luminosity profile.

We also obtain the radial variation of the $\alpha$-parameter values (Fig. 18) by scaling the PN number density individually in each elliptical bin, which shows an increase at larger radii. The increasing trend in the $\alpha$-parameter values is a direct consequence of the flattening of $\mu_{\mathrm{PN}}$, given the $B-V$ colour gradient of M 31. The radial variation of the $\alpha$-parameter values found by M06 also showed an slight increasing trend within the errors at larger radii. However, for the furthest radial bin in Fig. 18, the minor-axis surface brightness profile may be underestimating the light from the known inner halo substructures of M 31 primarily found along the disc major axis. This may also be contributing

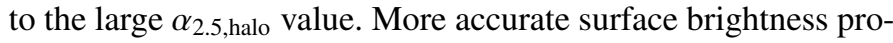
file measurements need to be performed in order to quantify the contribution of substructures to the minor-axis surface brightness profile of M31.

\section{Planetary nebula luminosity function}

For different galaxies, the generalised analytical formula for the PNLF introduced by Longobardi et al. (2013) is

$N(M)=c_{1} \mathrm{e}^{c_{2} M}\left(1-\mathrm{e}^{3\left(M^{*}-M\right)}\right)$,

where $c_{1}$ is a normalisation constant, $c_{2}$ is the slope at the faint end, and $M^{*}$ is the absolute magnitude of the LF bright cutoff. The Ciardullo et al. (1989) analytical LF is then a specific case of the generalised analytical formula with $c_{2}=0.307$ that reproduces their best fit to the PNLF of M31. Observations suggest that the slope described by the parameter $c_{2}$ is correlated with the star formation history of the parent stellar population (Ciardullo et al. 2004; Ciardullo 2010; Longobardi et al. 2013; Hartke et al. 2017). In order to ascertain the robustness of the morphology of the PNLF at magnitude ranges not reached before, we investigate the M 31 PNLF from our survey with different independent methods.

\subsection{PNLF of M31}

The PNLF is corrected for detection completeness and also for selection completeness due to the colour and point-like selection, detailed in Sect. 3.6. Figure 19 shows the PNLF of M 31 for all the PNe identified by our survey, fitted by the generalised analytical formula with $c_{2}=0.279 \pm 0.024$, which agrees well

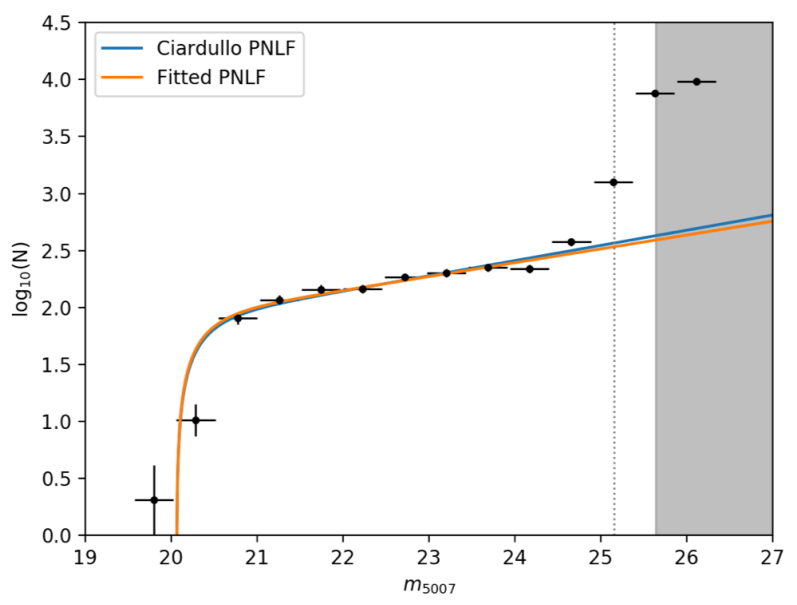

Fig. 19. Completeness-corrected PNLF for the whole catalogue of M 31 $\mathrm{PNe}$ is shown fitted by both the generalised analytical formula (in orange) and the Ciardullo et al. (1989) analytical LF (in blue). The region beyond the limiting magnitude of the shallowest field (Field\# 33_4) is shown in grey. The grey dotted line shows the $90 \%$ completeness limit of the shallowest field.

with that previously found by Ciardullo et al. (1989). Only the data corresponding to $m_{5007}<24$ are considered for the fit. The bright cut-off remains consistent with the known value of $M^{*}$. The faint end of the PNLF shows a rise with respect to the fitted function. This rise is seen at $m_{5007}>24.5$ in all the fields of the survey. This rise was not seen by M06 whose sample was photometrically complete to a brighter magnitude $\left(m_{5007}=23.5\right)$.

\subsection{Cumulative PNLF}

The M31 PNe can be fitted to a cumulative luminosity function (e.g., Peña et al. 2007, for NGC 3109) to avoid potential histogram binning considerations such as the bin size, limits, or the position of the first bin. However, some important features of the canonical PNLF (such as the dip) could be lost. Rodríguez-González et al. (2015) presented the Cumulative PNLF corresponding to the analytical PNLF described by Ciardullo et al. (1989). The Cumulative PNLF for the generalised PNLF is presented as follows:

$$
\begin{gathered}
I(M)=c_{1} \mathrm{e}^{c_{2} M}\left[\frac{1}{c_{2}} \mathrm{e}^{c_{2} M}+\frac{1}{3-c_{2}} \mathrm{e}^{3\left(M^{*}+\mu\right)-\left(3-c_{2}\right) M}\right. \\
\left.-\left(\frac{1}{c_{2}}+\frac{1}{3-c_{2}}\right) \mathrm{e}^{c_{2}\left(M^{*}+\mu\right)}\right] .
\end{gathered}
$$

Figure 20 shows the cumulative PNLF of M 31 for all the PNe identified by our survey, fitted by the generalised analytical formula with $c_{2}=0.257 \pm 0.011$, which agrees well with that found from the canonical PNLF, thereby corroborating that the effect histogram binning considerations on the canonical PNLF is negligible. Only the data corresponding to $m_{5007}<24$ are considered for the fit. The faint end of the cumulative PNLF also shows the rise with respect to the fitted function.

\subsection{Two-mode PNLF}

The rise at the faint end of the PNLF may be an indication that there are two PN populations, one dominating the brighter end and a second one at the fainter end. Thus, we fit the observed cumulative PNLF with two modes similar 


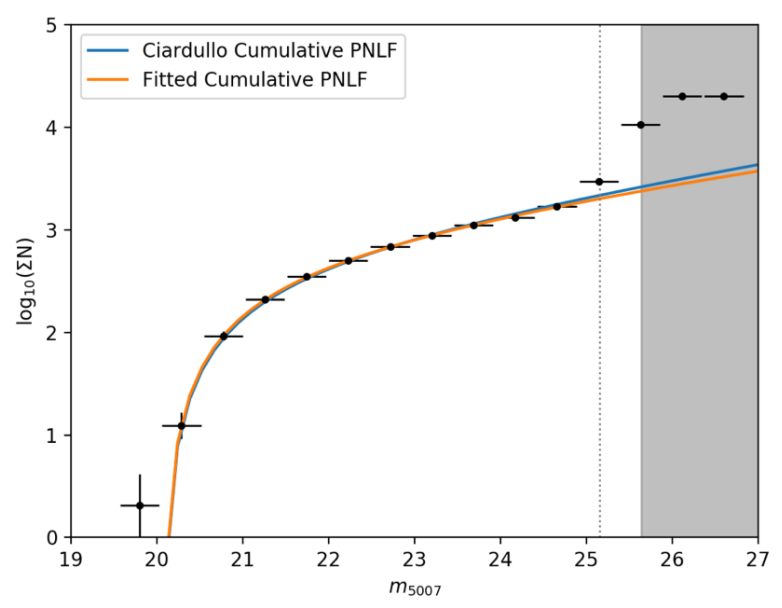

Fig. 20. Completeness-corrected cumulative PNLF (binned for visual clarity) for the whole catalogue of M 31 PNe is shown fitted by both the generalised analytical formula for the cumulative PNLF (in orange) and the cumulative PNLF corresponding to the Ciardullo et al. (1989) analytical formula (in blue). The region beyond the limiting magnitude of the shallowest field (Field\# 33_4) is shown in grey. The grey dotted line shows the $90 \%$ completeness limit of the shallowest field.

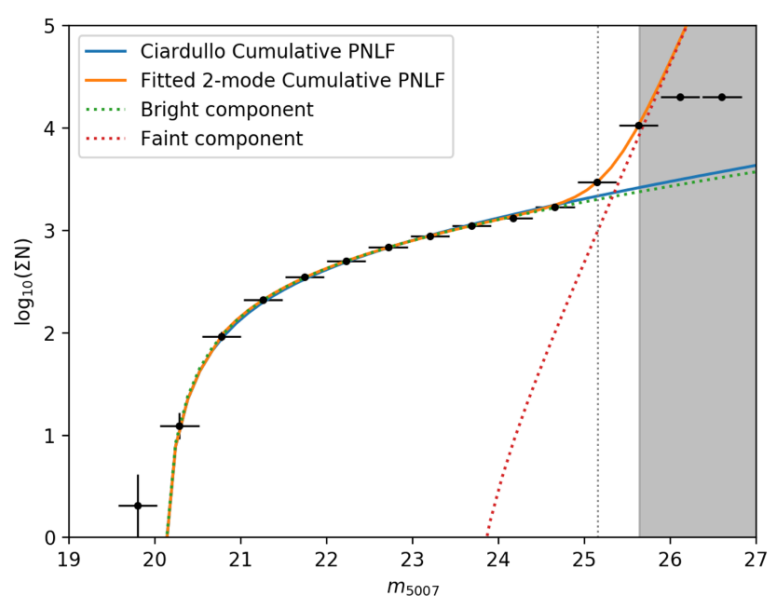

Fig. 21. Completeness-corrected cumulative PNLF (binned for visual clarity) for the whole catalogue of M31 PNe. It is fitted with two modes of the generalised analytical formula for the cumulative PNLF (in orange) with one component dominating the brighter end (in green) and another dominating the faint end (in red). The cumulative PNLF corresponding to the Ciardullo et al. (1989) analytical formula (in blue) is also shown. The region beyond the limiting magnitude of the shallowest field (Field\# 33_4) is shown in grey. The grey dotted line shows the $90 \%$ completeness limit of the shallowest field.

to Rodríguez-González et al. (2015). The two-mode PNLF is defined as $I_{2 m}(M)=I_{b}(M)+I_{f}(M)$, where $I_{b}(M)$ is the cumulative function for the generalised PNLF set to parameters found in the previous section, which accurately represents the observed PNLF before the rise at the faint end, and $I_{f}(M)$ is a cumulative function for the generalised PNLF considered with free parameters, $c_{f 1}, c_{f 2}$, and $M_{f}^{*}$, which are the normalisation constant, slope, and bright cut-off of a possible second faint $\mathrm{PNe}$ population. Rodríguez-González et al. (2015) had included an additional magnitude cut-off in their definition of the two-mode PNLF where the contribution of the brighter mode was set to zero at this magnitude and only the faint-end contribution was present. We do not include this magnitude cut-off because any $\mathrm{PN}$ population, such as the one dominating in the brighter end,

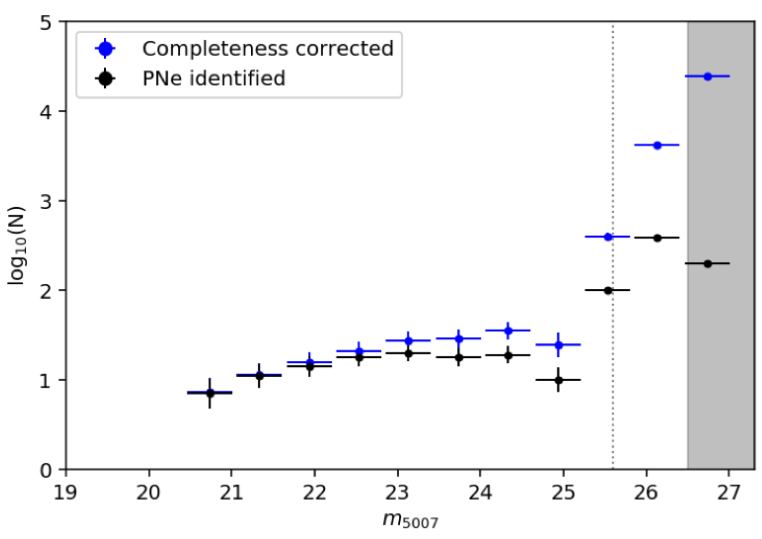

Fig. 22. For the deepest field (Field\# 35_4), the observed number of $\mathrm{PNe}$ in each magnitude bin (in black) and the completeness corrected number (in blue). The region beyond the limiting magnitude of this field is shown in grey. The grey dotted line indicates the $90 \%$ completeness limit of this field.

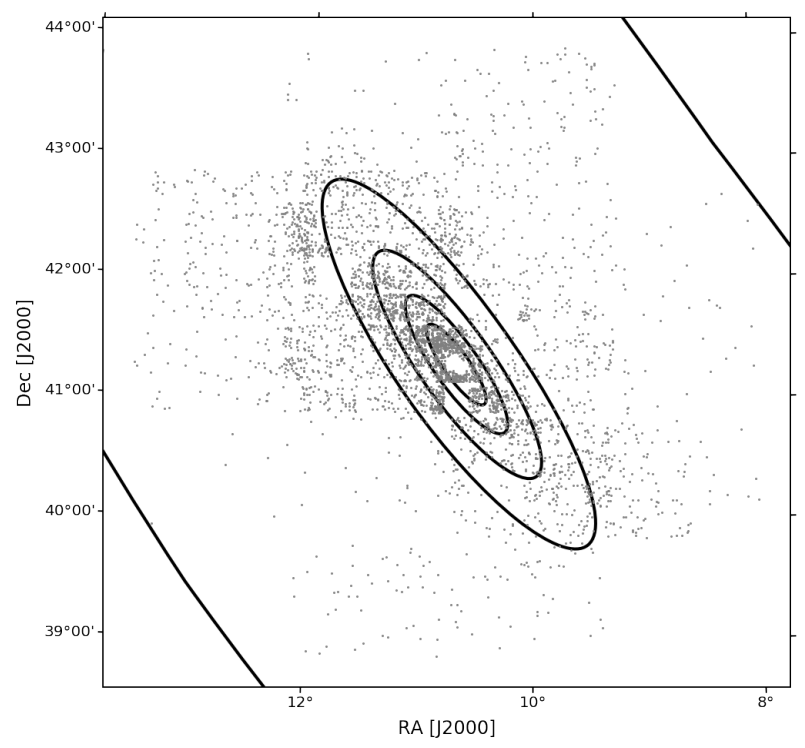

Fig. 23. Same as Fig. 16, but with five elliptical bins.

should have a contribution down to $\sim 8$ mag below the bright cut-off (Buzzoni et al. 2006). The two-mode fit of the observed PNLF is shown in Fig. 21. For the second possible PNe population, we find $c_{f 2}=4.4 \pm 0.1$ and $M_{f}^{*}=-1 \pm 0.3$. Thus, the observed cumulative PNLF may have a second PN population with a much steeper slope and very different bright cut-off.

\subsection{PNLF of the deepest field}

The deepest field in our survey is Field\# 35_4, which has a limiting magnitude at $m_{5007}=26.4$ and is $90 \%$ complete at $m_{5007}=$ 25.6. The PNLF for the PNe observed in this field is shown in Fig. 22 both before and after completeness correction. We note that the rise in the PNLF at $m_{5007}>25$ is present in the observed PNLF within the $90 \%$ completeness limit. Thus, the rise of the PNLF is a physical property of the observed PN sample.

\subsection{Radial variation of PNLF}

We divide the PNe spatially into five elliptical bins (Fig. 23) similar to Fig. 16. The PNLF corresponding to each bin is shown in 

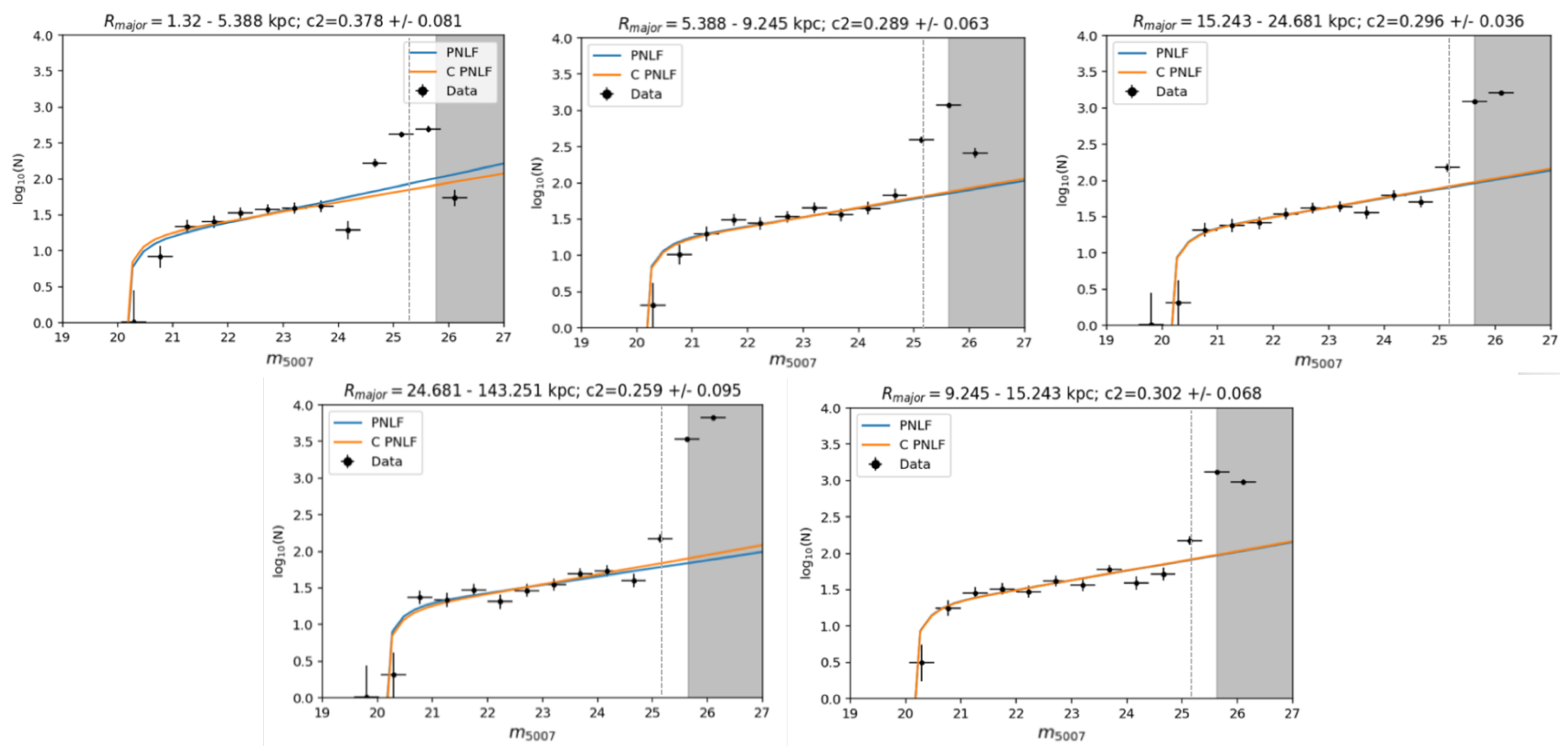

Fig. 24. Completeness-corrected PNLF for each elliptical bin fitted by both the generalised analytical formula (in blue) and the Ciardullo et al. (1989) analytical LF (in orange). The projected major-axis elliptical radii covered by each bin is indicated along with the fitted slope of the PNLF. The region beyond the limiting magnitude of the shallowest field (Field\# 33_4) is shown in grey. The grey dotted line shows the 90\% completeness limit of the shallowest field.

Fig. 24. Only the data corresponding to $m_{5007}<24$ are considered to fit each PNLF. We observe that the rise in the PNLF remains invariant as we go radially outwards indicating that the rise is ubiquitous throughout the surveyed area, and is not a function of photometry in crowded areas. Pastorello et al. (2013) identified $\mathrm{PNe}$ in the high metallicity nuclear region within $80 \mathrm{pc}$ of the centre of M31 with HST and SAURON data. They found a PNLF with a paucity of bright PNe within $\sim 1$ mag of the bright cut-off. We find that the bright cut-off remains invariant as we go radially outwards, but this is expected because the median metallicity of the disc and inner halo stars is largely uniform as the metal-poor inner halo is colligated with stars associated with the more metal-rich substructures (Ibata et al. 2014). We note that the PNLF corresponding to the innermost bin, covering part of the bulge of M 31 which is mostly saturated in our survey, has a steeper fitted slope as expected from PNe corresponding to an older parent stellar population (Longobardi et al. 2013).

\subsection{PNLF of PHAT-matched PNe}

Figure 25 shows the PNLF of the PHAT-matched PNe. The faint end of the PNLF still shows a rise even for the conservatively selected "likely" PNe. This indicates that the colour and magnitude of faint $\mathrm{PNe}$ are consistent with those of the bright PNe.

\subsection{Comparison with other deep PN surveys in Local Group galaxies}

We compare the shape of the M31 PNLF with that of other surveys in different galaxies that sample similar magnitude intervals from the bright cut-off. A catalogue of PNe with $m_{5007}$ down to $\sim 10$ mag below the bright cut-off of the PNLF is available for the Large Magellanic Cloud (LMC) from Reid \& Parker (2010). This catalogue is not completeness corrected as the $m_{5007}$ magnitudes are estimated from spectroscopy but is expected to be largely complete to $\sim 6 \mathrm{mag}$ below the bright cut-off, i.e. the magnitude interval covered by our survey of PNe in M31. Another

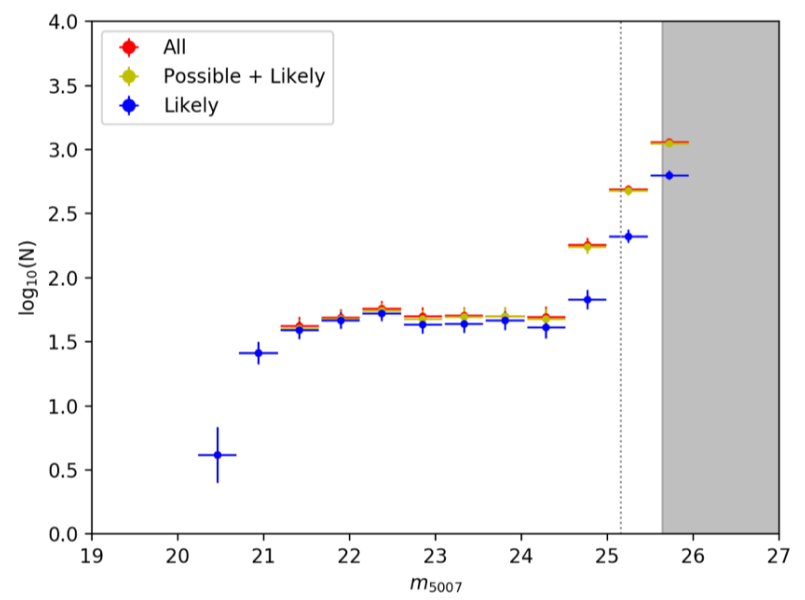

Fig. 25. Completeness-corrected PNLF for PHAT-matched PNe is shown for the "likely" (blue), "possible + likely" (yellow) and "possible + likely + unlikely" (red) cases. The region beyond the limiting magnitude of the shallowest field (Field\# 33_4) is shown in grey. The grey dotted line shows the $90 \%$ completeness limit of the shallowest field.

catalogue of PNe is available for the Small Magellanic Cloud (SMC) from Jacoby \& De Marco (2002) with $m_{5007}$ down to $\sim 8$ mag below the bright cut-off. It is also not corrected for completeness, but has significantly fewer PNe than the LMC and may suffer from significant completeness issues $\sim 6$ mag below the bright cut-off. A catalogue of PNe identified in the MW bulge is also available (Kovacevic et al. 2011), and assuming that all their PNe are at a constant $8 \mathrm{kpc}$ distance (Majaess 2010), i.e. the average distance to the MW bulge, a PNLF may be constructed. The distance approximation may be inaccurate, and completeness information is also unavailable.

Considering the LMC, SMC, and MW bulge PNe at the distance of M 31 normalised for the number of PNe in M 31, we can compare the shape of their PNLF with that of M 31 (Fig. 26). We note that the difference in the bright cut-off is expected from the 


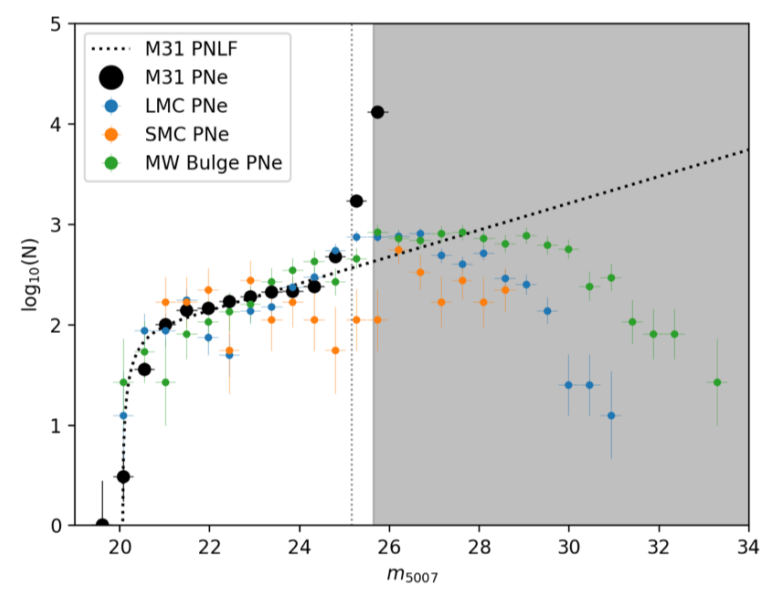

Fig. 26. Completeness-corrected PNLF for the whole catalogue of M 31 PNe (black points) is shown fitted by the Ciardullo et al. (1989) analytical LF (black dotted line). The LMC (Reid \& Parker 2010), SMC (Jacoby \& De Marco 2002), and MW Bulge PNe (Kovacevic et al. 2011) shifted to the distance of M 31 are shown in blue, orange, and green, respectively. The region beyond the limiting magnitude of the shallowest field (Field\# 33_4) is shown in grey. The grey dotted line shows the $90 \%$ completeness limit of the shallowest field.

difference in metallicity between the three galaxies. The dip in the PNLF seen for both the LMC and the SMC, albeit at different magnitudes, is not seen for M31. The MW bulge PNe seem to show a different slope, but no rise is evident. However, we cannot disregard the possibility that a dip or a rise at the faint end of the PNLF may be seen pending accurate distance determination. The rise at the faint end of the PNLF of M31 is much steeper than any of the others.

\section{Discussion}

\subsection{Possible sources of contamination}

As discussed in Sect. 3.4, up to 50 continuum sources may contaminate our PNe catalogue, most of which should be fainter than $m_{5007}=25$. This is also corroborated for the PHAT counterparts where $\sim 3 \%$ of the matched PNe may actually be stellar contaminants. The contamination from Milky Way (MW) halo and disc PNe is negligible as there are no MW halo PNe at $m_{5007}>20.5$ (Kovacevic et al. 2011). Background galaxies at redshift $z=0.345$ that are [OII] $3727 \AA$ emitters can be another source of contamination, but they are not known to have $\mathrm{EW}_{\text {obs }}>95 \AA$ (Colless et al. 1990; Hammer et al. 1997; Hogg et al. 1998). Our colour selection thus renders their contamination negligible.

$\mathrm{H}$ II regions are also bright in the [O III] $5007 \AA$ line and are present in the same region of the CMD as PNe. However, $\mathrm{H}_{\text {II }}$ regions appear extended at the distance of M31 and with the photometric quality of our survey, we are able to significantly limit their contamination as seen in Sect. 4. Some compact H II regions, especially in the disc of M 31, may still contaminate our survey. Of the $253 \mathrm{H}$ in regions identified by Sanders et al. (2012) later from spectroscopy, M06 had classified 101 of them as PNe. We find only 15 of these in our survey as PNe, thereby corroborating the excellent photometric quality of our survey. Other [O III] $5007 \AA$ sources like SySts may also mimic PNe and contaminate our survey. We investigate in the following whether the contribution from SySts is responsible for the two-mode PNLF investigated in Sect. 6.3.

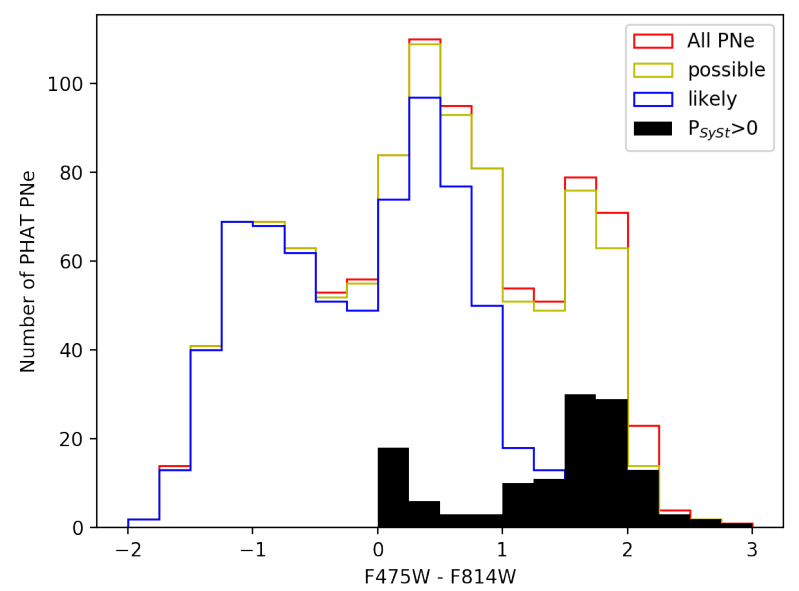

Fig. 27. Histogram for PHAT-matched PNe is shown for the "likely" (blue), "possible + likely" (yellow) and "possible + likely + unlikely" (red) cases. The histogram of possible SySts are shown in black.

\subsection{Symbiotic star contribution to PNLF}

SySts are among the longest orbital period interacting binaries, consisting of an evolved cool giant and an accreting, hot, luminous companion (usually a white dwarf) surrounded by a dense ionised nebula. Depending on the nature of the cool giant, there are two main classes of SySts: the S-types (stellar), which are normal $\mathrm{M}$ giants with orbital periods of the order of a few years, and the D-types (dusty), which contain Mira variable primaries surrounded by warm dust with orbital periods of a decade or longer. Mikołajewska et al. (2014) found 31 confirmed Systs in M 31, 10 of which had unambiguous PHAT counterparts. Of these, 9 were S-types and their spectra show that the continuum emission is high in the F814W filter. Mikołajewska et al. (2014) utilised this to identify the PHAT counterpart of their SySt as the PHAT source that had the brightest F814W mag in their $0.75^{\prime \prime}$ search radius. It is also seen that the F475W-F814W colour of these SySt generally have a high positive value which can be used to distinguish them from PNe.

We estimate the probability that each of our PHAT-matched PN is a SySt by checking how close it was in F814W mag $\left(\mathrm{F} 814 \mathrm{~W}_{\mathrm{PN}}\right)$ to the brightest $\mathrm{F} 814 \mathrm{~W}$ mag source $\left(\mathrm{F} 814 \mathrm{~W}_{\text {high }}\right)$ in our search radius, and also if it had a colour excess in F814W with respect to $\mathrm{F} 475 \mathrm{~W}$. The probability, $P_{\text {SySt }}$, is given by the following formula:

$P_{\mathrm{SySt}}=\frac{\mathrm{F} 814 \mathrm{~W}_{\mathrm{PN}}-\overline{\mathrm{F} 814 \mathrm{~W}}}{\mathrm{~F} 814 \mathrm{~W}_{\mathrm{high}}-\overline{\mathrm{F} 814 \mathrm{~W}}} \times P_{\mathrm{col}}$.

Here $\overline{\mathrm{F} 814 \mathrm{~W}}$ is the mean F814W mag of all sources in the search radius and $P_{\text {col }}$ is the colour excess check, which equals 1 if F475W-F814W $>1$ (most of the Mikołajewska et al. 2014 SySt have this), $1 / 2$ if $0<$ F475W-F814W $<1$ (some of the confirmed PNe have this), and 0 otherwise. PHAT-matched PN with F814W below the F814W are set to have a $P_{\text {SySt }}=0$. We find that there is a systematic overestimation of $P_{\text {SySt }}$ since PHATmatched PNe just above their $\overline{\mathrm{F} 814 \mathrm{~W}}$ would still be assigned a small value of $P_{\text {SySt }}$. We thus update the $P_{\text {SySt }}$ by subtracting the mean of the probability, $\overline{P_{\text {SySt }}}$, from each source and setting those with negative values to zero. We find that most PHAT-matched PNe with a probability of being a SySt are clustered around a high F475W-F814W colour (Fig. 27). Removing the contribution of SySts from the PNLF (Fig. 28), it is evident 


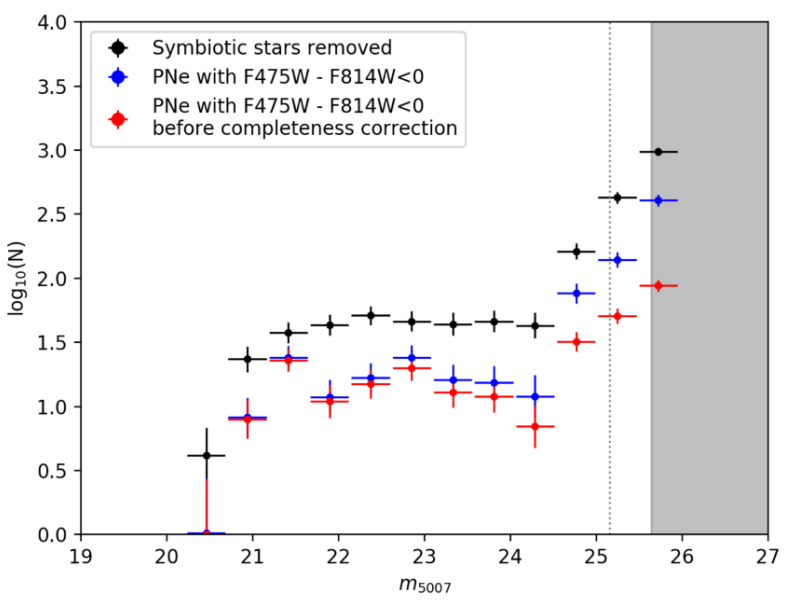

Fig. 28. Completeness-corrected PNLF for PHAT-matched PNe with the contribution from SySts removed is shown in black. The completeness-corrected PNLF for only those PNe with F475WF814W $<0$ is shown in blue. The PNLF for the observed PHATmatched $\mathrm{PNe}$ with $\mathrm{F} 475 \mathrm{~W}-\mathrm{F} 814 \mathrm{~W}<0$ is shown in red. The region beyond the limiting magnitude of the shallowest field (Field\# 33_4) is shown in grey. The grey dotted line shows the $90 \%$ completeness limit of the shallowest field.

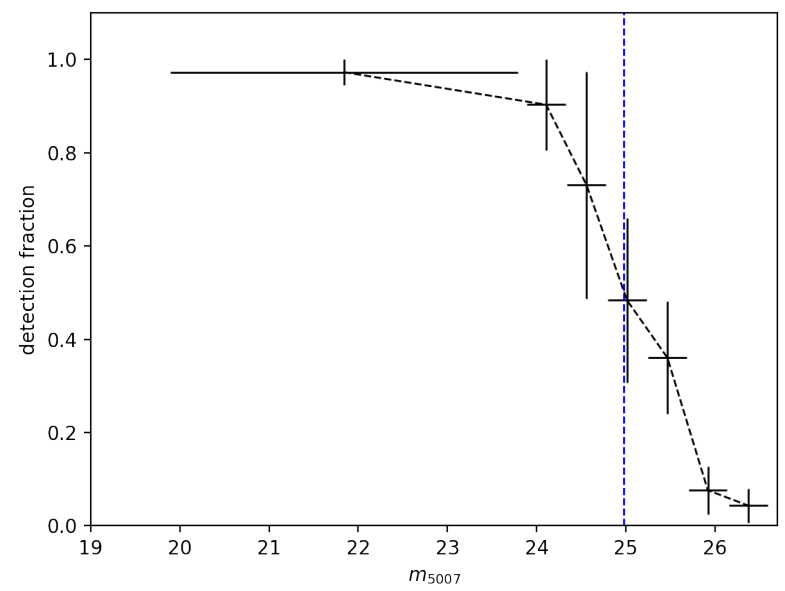

Fig. 29. Fraction of $\mathrm{PNe}$ targeted with spectroscopic observations where a narrow [O III] $5007 \AA$ emission line was detected. The uncertainty in detection fraction is the binomial proportion confidence-interval of observed PNe in any magnitude bin obtained using the Wilson score interval method (Wilson 1927). The blue dashed line shows the $50 \%$ detection limit of the spectroscopic follow-up.

that the rise at the faint end of the PNLF cannot be explained by SySts. Figure 28 also shows the PNLF for only those PHATmatched $\mathrm{PNe}$ with $\mathrm{F} 475 \mathrm{~W}-\mathrm{F} 814 \mathrm{~W}<0$ to show that the rise in the PNLF is not dependent on the F475W-F814W colour. Continuum stars are unlikely to have F475W-F814W $<0$ and considering that we limit contamination from $\mathrm{H}$ II regions, the sources with $\mathrm{F} 475 \mathrm{~W}-\mathrm{F} 814 \mathrm{~W}<0$ are most likely genuine PNe. The rise in the observed PNLF is indeed visible, even prior to completeness correction.

D-type SySts do not show an excess in the F814W filter. Thus, in our analysis a D-type SySt cannot be distinguished from a PNe. Even so, their numbers are expected to be far lower than that of a S-type SySt and so there should not be any significant number of D-type SySts plaguing our data. Mikołajewska et al. (2014) had one D-type SySt in the PHAT footprint, which we indeed misidentify as a PNe in our study.

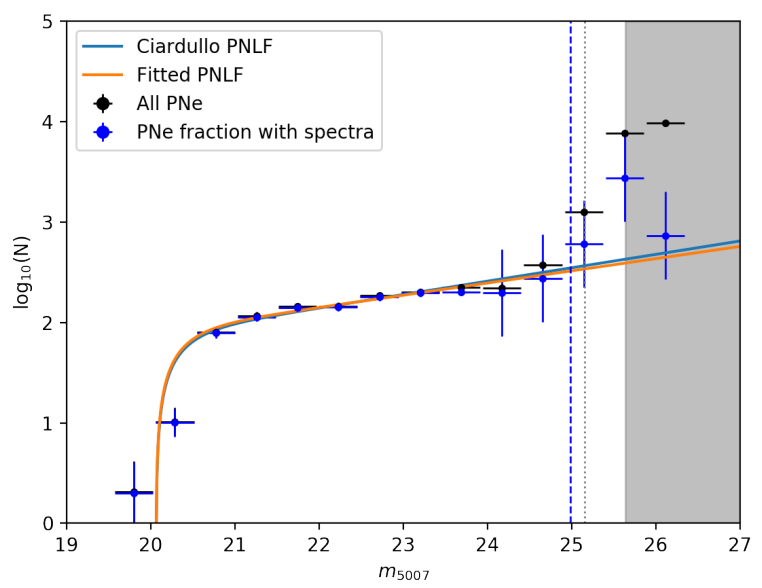

Fig. 30. Completeness-corrected PNLF for all PNe (in black) fitted by both the generalised analytical formula for the cumulative PNLF (in orange) and the cumulative PNLF corresponding to the Ciardullo et al. (1989) analytical formula (in blue). The completeness-corrected PNLF accounting for the detection fraction from spectroscopy is shown in blue. The region beyond the limiting magnitude of the shallowest field (Field\# 33_4) is shown in grey. The grey dotted line shows the $90 \%$ completeness limit of the shallowest field. The blue dashed line shows the $50 \%$ detection limit of the spectroscopic follow-up.

\subsection{Spectroscopically confirmed PNe and the faint end of the PNLF}

A spectroscopic follow-up of a complete subsample of the M31 $\mathrm{PNe}$ was carried out with the Hectospec multifibre positioner and spectrograph on the Multiple Mirror Telescope (MMT; Fabricant et al. 2005). Observations were made on September 15,2018 , and October 10, 2018, with an exposure time of $9000 \mathrm{~s}$ each, and also on December 4, 2018, with an exposure time of $3600 \mathrm{~s}$. The Hectospec 270 gpm grating was used and provided spectral coverage from 3650 to $9200 \AA$ at a resolution of $\sim 5 \AA$. Each Hectospec fibre subtends $1.5^{\prime \prime}$ on the sky. The fibres were placed on PNe candidates to maximise the observation of bright PNe, obtain their LOSV, and determine their chemical abundances. Of the 343 PNe candidates observed in M31, 129 had confirmed detection of the [O III] 4959/5007 $\AA$ emission lines. The [O III] $5007 \AA$ emission line was detected in all cases with a signal-to-noise ratio higher than 8 . All of them also had the $\mathrm{H} \alpha$ line present. Since our original PNe sample is largely devoid of H II regions, these observed sources are expected to be PNe. Details of the spectroscopy confirming them as PNe, along with their LOSV distribution and chemical abundances will be presented in a forthcoming paper (Bhattacharya et al., in prep.). The fraction of PNe detected as a function of magnitude is shown in Fig. 29.

The spectroscopic follow-up shows that all the PNe candidates observed were confirmed as $\mathrm{PNe}$, from the presence of [O III] 4959/5007 $\AA$ and $\mathrm{H} \alpha$ emission lines, down to $24.5 \mathrm{mag}$, but only a fraction of the targeted PNe candidates at fainter magnitudes could be confirmed. While the required emission lines may not have been detected due to a low signal-to-noise ratio in the spectra for the faint targeted PNe candidates, it is also possible that those faint sources are continuum contaminants or background galaxies instead. Making the conservative assumption that all such sources are not PNe, we can modify the PNLF to account for this behavior. Since the rise in the PNLF is seen at different radii at about the same extent, we can compute the expected PNLF for the fraction of PNe that are conservatively confirmed spectroscopically, as shown in Fig. 30. The PNLF is 
still consistent with the rise at the faint end of the PNLF although the uncertainty in the fraction with spectral confirmation is large.

\subsection{Summary of the observational evidence for the rise at the faint end of the PNLF}

Our investigation of the morphology of the PNLF is carried out at different positions and radial distances, and considering the effects of possible contaminants. The rise at the faint end of the PNLF is observed not only in the PNLF for the whole survey but also in individual fields, including the deepest field discussed in Sect. 6.4. In this field, it is already visible for the observed PNe even before completeness correction.

The rise at the faint end of the PNLF occurs from $m_{5007} \sim$ $24.5-25$ and is present at varying radial distances from the centre of M 31 (Sect. 6.5). If the rise at the faint end of the PNLF was due to compact $\mathrm{H}$ In regions, it would be largely present only in the disc of M 31 and not in all radial elliptical bins. In the survey region in common with $H S T$ data from PHAT, we also see the rise in the PNLF for those PNe with reliable PHAT counterparts (Sect. 6.6). The rise is especially evident when looking at the PNLF from PHAT-matched PNe with F475W-F814W $<0$, a region in colour occupied almost exclusively by $\mathrm{PNe}$, even prior to completeness correction (Fig. 28). The spectroscopic followup also shows that the rise at the faint end of the PNLF is seen in confirmed $\mathrm{PNe}$, albeit with large errors.

This observational evidence means that it is reasonable to believe that the rise in the PNLF is indeed physical and associated with the PN population and their parent stars. Rodríguez-González et al. (2015) fitted the two-mode PNLF for NGC 6822 (an irregular galaxy in the local group) and from its star formation history they show that the two modes of the PNLF may correspond to PNe associated with the two episodes of star formation, with the older parent stellar population corresponding to the fainter PNe. It is possible that the second PN population in M 31 is associated with an older stellar population. With data from the PHAT survey, Williams et al. (2017) found that there was a burst of star formation 2-4 Gyr ago throughout the M31 stellar disc. Bernard et al. (2015) studied individual HST fields associated with the disc and inner halo substructures of M 31 to find that all the fields show a burst of star formation $\sim 2 \mathrm{Gyr}$ ago even though most of the stars in the M31 outer disc formed $>8 \mathrm{Gyr}$ ago. Since the slope of the PNLF associated with PNe belonging to an older stellar population is seen to be steeper (Sect. 6.3), it is possible that the steep rise at the faint end of the PNLF is caused by PNe associated with the older population while the PNe associated with the $\sim 2 \mathrm{Gyr}$ old burst of star formation populate the brighter magnitudes of the PNLF.

Another possible reason for the rise in the faint end of the PNLF could be a change in opacity of the nebula of the PNe. The PNe occupying the bright end of the PNLF could be more opaque, with the nebula more efficiently reprocessing the ionising flux of the central star into [O III] $5007 \AA$ while the faint end may be populated by the more transparent PNe. The significant dependence of the PNLF on the considerations of the nebular transparency is also evident in the models by Gesicki et al. (2018). This scenario has previously been invoked to describe the dip in the PNLF seen in the SMC (Jacoby \& De Marco 2002).

\section{Conclusions}

We present a 16 square degree survey in the disc and inner halo of M 31 with the MegaCam at the CFHT using the narrow- band [O III] $5007 \AA$ filter and the broad-band $g$ filter. We identify point-lke PNe from the colour excess between the narrow-band and broad-band images down to a limiting magnitude between $m_{5007}=25.64$ for the shallowest observed field to $m_{5007}=$ 26.4 for the deepest observed field. We obtain 4289 PNe, the largest sample outside the MW, of which 1099 were previously observed by M06. Using a simulated PN population, we are able to account for incompleteness. We find counterparts in PHAT for 1023 of our identified PNe in the PHAT footprint. We find no resolved H II regions and a very small fraction $(\sim 3 \%)$ of PNe that could be stellar contamination. The $\alpha$-parameter value obtained shows an increasing trend as we go further away from the centre of M31, but reasonably matches the value obtained by M06 for the central regions. The high value of the obtained $\alpha_{2.5 \text {, halo may }}$ be attributed to the bluer halo of M31 indicating that the stellar population in the halo, at radii larger than $\sim 10 \mathrm{kpc}$, may be different from that of the inner disc.

The PNLF of the whole sample is complete down to $\sim 5.5$ mag fainter than the bright cut-off and shows a significant rise at the faint end. The generalised analytical formula fitted to the completeness corrected PNLF, for magnitudes brighter than $m_{5007}<24$, returns parameters that are in agreement with those previously determined by Ciardullo et al. (1989). The rise at the faint end is seen at different elliptical radial distances from the centre and also in the PHAT-matched PNe implying that this rise is not associated with crowding. The rise is steeper than that seen in the LMC and the SMC. It is also not caused by contaminating sources like symbiotic stars, and seems to be a property of the parent population of the $\mathrm{PNe}$, evident from the comparison with PHAT. Early findings from the spectroscopic follow-up also show that the rise in the number of PNe at faint magnitudes is physical, albeit with large uncertainty. It is possible that the $\mathrm{PNe}$ in M 31 associated with the population created from the burst of star formation $\sim 2 \mathrm{Gyr}$ ago populate the brighter magnitudes of the PNLF, while those at the faint end are associated with the older population. It is also possible that the faint end is populated by PNe with more transparent nebulae ${ }^{7}$, while those with opaque nebulae populate the bright end.

Acknowledgements. SB and JH acknowledge support from the IMPRS on Astrophysics at the LMU Munich. We are grateful to the anonymous referee for the constructive comments that improved the manuscript. This research made use of IRAF, distributed by the National Optical Astronomy Observatory, which is operated by the Association of Universities for Research in Astronomy (AURA) under a cooperative agreement with the National Science Foundation. This research made use of Astropy, a community-developed core Python package for Astronomy (Astropy Collaboration 2013); Numpy (Oliphant 2015); and Matplotlib (Hunter 2007). This research also made use of NASA's Astrophysics Data System (ADS).

\section{References}

Arnaboldi, M., Freeman, K. C., Mendez, R. H., et al. 1996, ApJ, 472, 145 Arnaboldi, M., Freeman, K. C., Gerhard, O., et al. 1998, ApJ, 507, 759 Arnaboldi, M., Aguerri, J. A. L., Napolitano, N. R., et al. 2002, AJ, 123, 760 Arnaboldi, M., Freeman, K. C., Okamura, S., et al. 2003, AJ, 125, 514 Astropy Collaboration (Robitaille, T. P., et al.) 2013, A\&A, 558, A33 Barmby, P., Ashby, M. L. N., Bianchi, L., et al. 2006, ApJ, 650, L45 Bernard, E. J., Ferguson, A. M. N., Chapman, S. C., et al. 2015, MNRAS, 453, L113

Bertin, E., \& Arnouts, S. 1996, A\&AS, 117, 393

Bertin, E., Mellier, Y., Radovich, M., et al. 2002, in Astronomical Data Analysis Software and Systems XI, eds. D. A. Bohlender, D. Durand, \& T. H. Handley, 281, 228

Bonnarel, F., Fernique, P., Bienaymé, O., et al. 2000, A\&AS, 143, 33

https://ui . adsabs.harvard.edu 
Boulade, O., Charlot, X., Abbon, P., et al. 2003, in Instrument Design and Performance for Optical/Infrared Ground-based Telescopes, eds. M. Iye, \& A. F. M. Moorwood, 4841, 72

Buzzoni, A., Arnaboldi, M., \& Corradi, R. L. M. 2006, MNRAS, 368, 877 Ciardullo, R. 2010, PASA, 27, 149

Ciardullo, R., \& Jacoby, G. H. 1992, ApJ, 388, 268

Ciardullo, R., Jacoby, G. H., Ford, H. C., \& Neill, J. D. 1989, ApJ, 339, 53

Ciardullo, R., Feldmeier, J. J., Jacoby, G. H., et al. 2002, ApJ, 577, 31

Ciardullo, R., Durrell, P. R., Laychak, M. B., et al. 2004, ApJ, 614, 167

Ciardullo, R., Gronwall, C., Adams, J. J., et al. 2013, ApJ, 769, 83

Coccato, L., Gerhard, O., Arnaboldi, M., et al. 2009, MNRAS, 394, 1249

Colless, M., Ellis, R. S., Taylor, K., \& Hook, R. N. 1990, MNRAS, 244, 408

Cortesi, A., Arnaboldi, M., Coccato, L., et al. 2013, A\&A, 549, A115

Courteau, S., Widrow, L. M., McDonald, M., et al. 2011, ApJ, 739, 20

Dalcanton, J. J., Williams, B. F., Lang, D., et al. 2012, ApJS, 200, 18

Davis, B. D., Ciardullo, R., Jacoby, G. H., Feldmeier, J. J., \& Indahl, B. L. 2018 ApJ, 863, 189

Douglas, N. G., Arnaboldi, M., Freeman, K. C., et al. 2002, PASP, 114, 1234

D’Souza, R., \& Bell, E. F. 2018, Nat. Astron., 2, 737

Fabricant, D., Fata, R., Roll, J., et al. 2005, PASP, 117, 1411

Fang, X., García-Benito, R., Guerrero, M. A., et al. 2015, ApJ, 815, 69

Fang, X., García-Benito, R., Guerrero, M. A., et al. 2018, ApJ, 853, 50

Ferguson, A. M. N., \& Mackey, A. D. 2016, in Tidal Streams in the Local Group and Beyond, eds. H. J. Newberg, \& J. L. Carlin, 420, 191

Gesicki, K., Zijlstra, A. A., \& Miller Bertolami, M. M. 2018, Nat. Astron., 2, 580

Hammer, F., Flores, H., Lilly, S. J., et al. 1997, ApJ, 481, 49

Hammer, F., Yang, Y. B., Wang, J. L., et al. 2018, MNRAS, 475, 2754

Hartke, J., Arnaboldi, M., Longobardi, A., et al. 2017, A\&A, 603, A104

Hartke, J., Arnaboldi, M., Gerhard, O., et al. 2018, A\&A, 616, A123

Henize, K. G., \& Westerlund, B. E. 1963, ApJ, 137, 747

Hernández-Martínez, L., \& Peña, M. 2009, A\&A, 495, 447

Hogg, D. W., Cohen, J. G., Blandford, R., \& Pahre, M. A. 1998, ApJ, 504, 622

Hui, X., Ford, H. C., Freeman, K. C., \& Dopita, M. A. 1995, ApJ, 449, 592

Hunter, J. D. 2007, Comput. Sci. Eng., 9, 90

Ibata, R. A., Lewis, G. F., McConnachie, A. W., et al. 2014, ApJ, 780, 128

Irwin, M. J., Ferguson, A. M. N., Ibata, R. A., Lewis, G. F., \& Tanvir, N. R. 2005, ApJ, 628, L105

Jacoby, G. H. 1980, ApJS, 42, 1
Jacoby, G. H. 1989, ApJ, 339, 39

Jacoby, G. H., \& De Marco, O. 2002, AJ, 123, 269

Jones, D., \& Boffin, H. M. J. 2017, Nat. Astron., 1, 0117

Kovacevic, A. V., Parker, Q. A., Jacoby, G. H., et al. 2011, MNRAS, 414, 860

Li, A., Li, Z., Dong, H., Fang, X., \& Xu, X. 2018, Res. Astron. Astrophys., 18, 140

Longobardi, A., Arnaboldi, M., Gerhard, O., et al. 2013, A\&A, 558, A42

Longobardi, A., Arnaboldi, M., Gerhard, O., \& Hanuschik, R. 2015, A\&A, 579, A135

Mackey, A. D., Huxor, A. P., Ferguson, A. M. N., et al. 2010, ApJ, 717, L11

Magnier, E. A., \& Cuillandre, J. C. 2004, PASP, 116, 449

Majaess, D. 2010, Acta Astron., 60, 55

Marmo, C., \& Bertin, E. 2008, in Astronomical Data Analysis Software and Systems XVII, eds. R. W. Argyle, P. S. Bunclark, \& J. R. Lewis, 394, 619 Martin, T. B., Drissen, L., \& Melchior, A.-L. 2018, MNRAS, 473, 4130

McConnachie, A. W., Irwin, M. J., Ibata, R. A., et al. 2009, Nature, 461, 66 McConnachie, A. W., Ibata, R., Martin, N., et al. 2018, ApJ, 868, 55 Méndez, R. H., Riffeser, A., Kudritzki, R. P., et al. 2001, ApJ, 563, 135

Merrett, H. R., Merrifield, M. R., Douglas, N. G., et al. 2006, MNRAS, 369, 120 Mikołajewska, J., Caldwell, N., \& Shara, M. M. 2014, MNRAS, 444, 586

Oke, J. B., \& Gunn, J. E. 1983, ApJ, 266, 713

Oliphant, T. E. 2015, Guide to NumPy, 2nd edn. (USA: CreateSpace Independent Publishing Platform)

Pastorello, N., Sarzi, M., Cappellari, M., et al. 2013, MNRAS, 430, 1219

Peña, M., Richer, M. G., \& Stasińska, G. 2007, A\&A, 466, 75

Pulsoni, C., Gerhard, O., Arnaboldi, M., et al. 2018, A\&A, 618, A94

Reid, W. A., \& Parker, Q. A. 2010, MNRAS, 405, 1349

Rodríguez-González, A., Hernández-Martínez, L., Esquivel, A., et al. 2015, A\&A, 575, A1

Sanders, N. E., Caldwell, N., McDowell, J., \& Harding, P. 2012, ApJ, 758, 133

Tempel, E., Tamm, A., \& Tenjes, P. 2010, A\&A, 509, A91

Teplitz, H. I., Malkan, M. A., Steidel, C. C., et al. 2000, ApJ, 542, 18

Veljanoski, J., Mackey, A. D., Ferguson, A. M. N., et al. 2014, MNRAS, 442, 2929

Veyette, M. J., Williams, B. F., Dalcanton, J. J., et al. 2014, ApJ, 792, 121

Walterbos, R. A. M., \& Kennicutt, Jr., R. C. 1988, A\&A, 198, 61

Williams, B. F., Lang, D., Dalcanton, J. J., et al. 2014, ApJS, 215, 9

Williams, B. F., Dolphin, A. E., Dalcanton, J. J., et al. 2017, ApJ, 846, 145

Wilson, E. B. 1927, J. Am. Stat. Assoc., 22, 209 


\section{Appendix A: Catalogue extraction}

\section{A.1. Flux calibration for $m_{5007}$}

The integrated flux, $F_{5007}$, from the [O III] $5007 \AA$ line is related to the $m_{5007}$ magnitude (Jacoby 1989) as

$m_{5007}=-2.5 \log F_{5007}-13.74$,

where the flux is in units of $\mathrm{erg} \mathrm{cm}^{2} \mathrm{~s}^{-1}$. The $A B$ magnitude (Oke \& Gunn 1983) relates to $F_{5007}$ through the MegaCam narrow-band [O III] filter characteristics as

$m_{\mathrm{n}}=-2.5 \log F_{5007}-2.5 \log \frac{\lambda_{\mathrm{c}^{2}}}{\Delta \lambda_{\mathrm{eff}} c}-48.59$,

where $\lambda_{\mathrm{c}}=5007 \AA$ and $\Delta \lambda_{\mathrm{eff}}=\Delta \lambda \times T$ for the on-band with $\Delta \lambda=102 \AA$ and effective transmission, $T=0.91$. The relation between the $A B$ and $m_{5007}$ mag, for this narrow-band filter, is thus determined (following Arnaboldi et al. 2003) to be

$m_{5007}=m_{\mathrm{n}}+2.27$

\section{A.2. Point spread function}

The point spread function (PSF) is the instrumental response to a point-like object. The degree of spreading of the PSF represents the quality of the instrument imaging. To determine the PSF, we first used the IRAF tasks daofind, daophot, and pstsel to respectively extract all the positions of detected sources, determine their magnitude, and select the brighter stars. We then used the IRAF task $p s f$, which allowed us to select the best stars to fit the PSF, and reject bad objects (e.g., saturated or located near an another source). The confirmed stars are used to calculate the PSF. The PSF is modelled with different luminosity profiles (e.g., Gaussian, Moffat function) and the best fit is selected using $\chi^{2}$ minimisation (goodness of fit). The best fit is a Moffat function (parameters in Table A.1) of the form

$f(r, \alpha, \beta)=\frac{\beta-1}{\pi \alpha}\left[1+\left(\frac{r}{\alpha}\right)^{2}\right]^{-\beta}$.

\section{A.3. Masking of noisy regions and CCD edges}

After identifying sources on the images, the image regions that were affected by dithering or saturation are masked on the onband and off-band images. This is carried out for each field. Dithering leads to different exposure depths at the image edges. Due to the combination of the 42 MegaCam CCDs, the same effect also affects columns at the borders of the individual CCDs. Additionally, in order to mask those regions with a high background value (e.g., due to charge transfer or saturated stars) we used the rms-background map created by SExtractor and create a pixel-mask with all values higher than three times the median background.

\section{A.4. Choice of aperture width}

After simulating sources on to the on-band, we determine the magnitude aperture most suited to recovering them. Figure A.1 shows that different SExtractor aperture widths (AW) of 15, 17, 19, and 21 pixels all recover the magnitudes well but an AW of 15 pixels can recover sources most accurately even for fainter sources.
Table A.1. Parameters for the best fitting PSF.

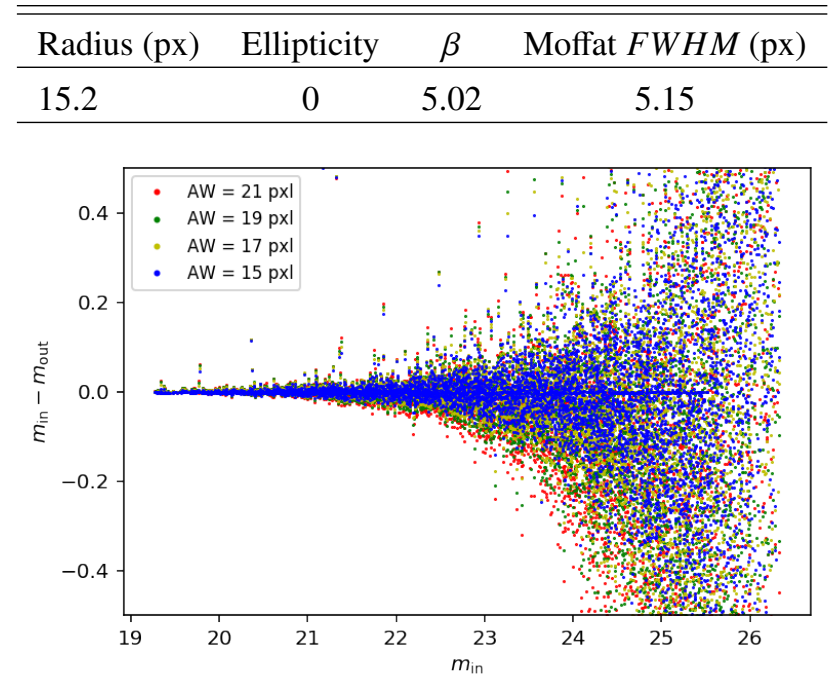

Fig. A.1. Recovery of the input magnitude of simulated sources using different SExtractor aperture widths, AW, for a single field.

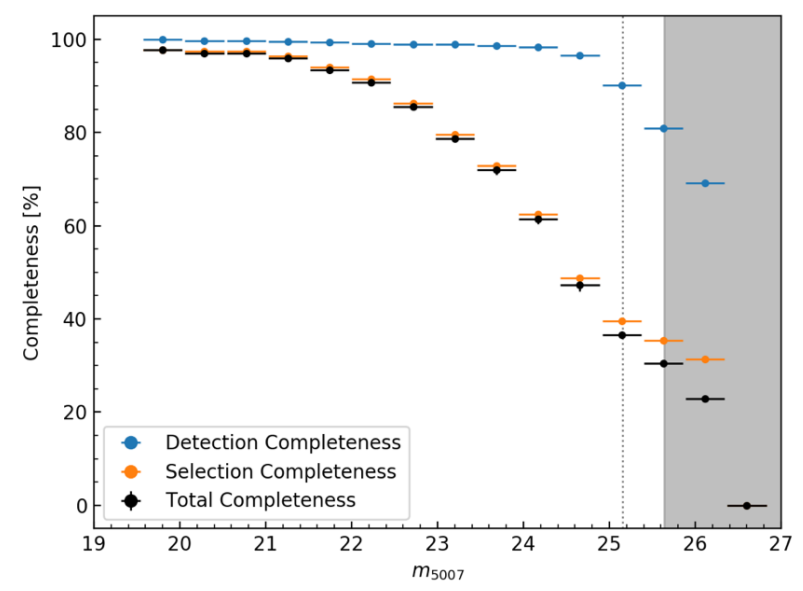

Fig. A.2. For the entire survey the number of $\mathrm{PNe}$ in each magnitude bin (in black), and the completeness corrected number (in blue) for a single field. The region beyond the limiting magnitude of the shallowest field (Field\# 33_4) is shown in grey. The grey dotted line shows the $90 \%$ completeness limit of the shallowest field.

\section{A.5. Completeness correction in the survey}

For the entire survey, the mean percentage total completeness is shown as a function of the $m_{5007}$ magnitude in Fig. A.2 in black, while the mean percentage detection completeness and percentage selection completeness are shown in blue and orange, respectively. It is clear that the completeness correction applied is dominated by the selection completeness, correcting for the number of genuine PNe missed due to our colour and point-like selection. The percentage completeness shown is only representative in Fig. A.2; the completeness varies in each field and the completeness correction is applied to PNe in each field separately.

\section{A.6. Detection check on background}

For each image, as a by-product of the source extraction, we obtain a background image. In order to check for spurious sources in any image, we run SExtractor on this background image with the parameters described in Sect. 3.1. The sources 

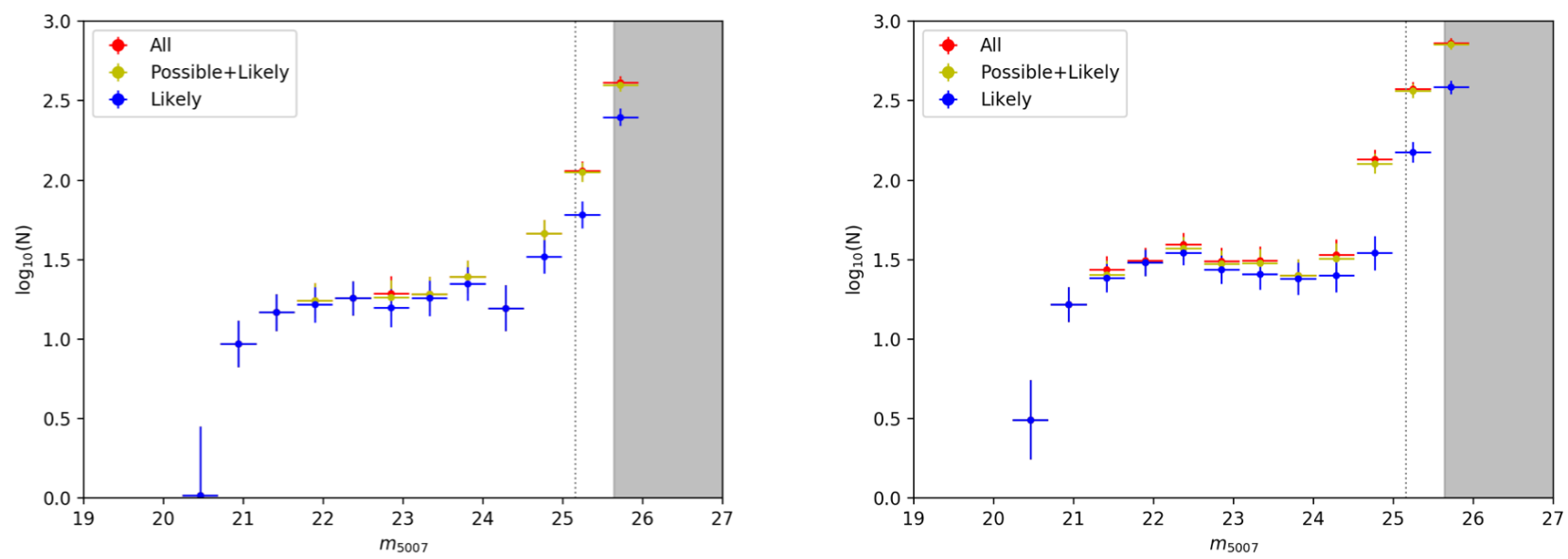

Fig. A.3. Completeness-corrected PNLF for PHAT-matched PNe within (left) and outside (right) 0.3 deg of the centre of M 31 is shown for the "likely" (blue), "possible + likely" (yellow), and "possible + likely + unlikely" (red) cases. The region beyond the limiting magnitude of the shallowest field (Field\# 33_4) is shown in grey. The grey dotted line shows the 90\% completeness limit of the shallowest field.

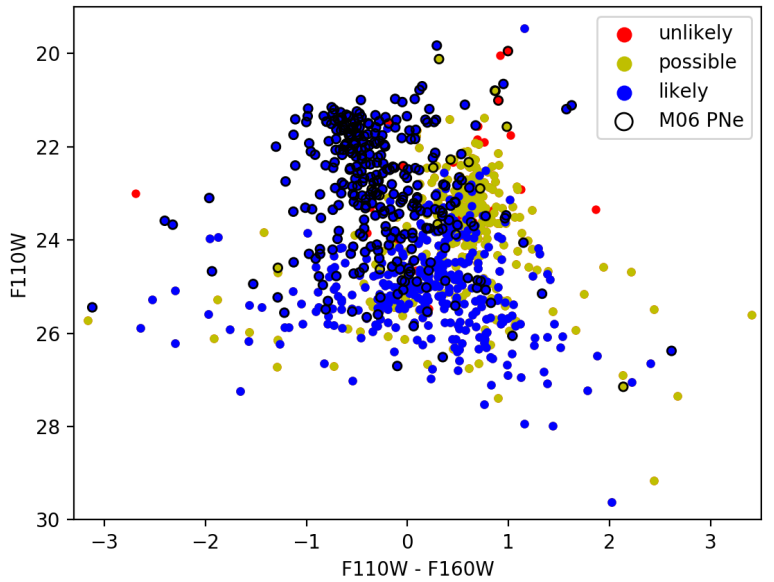

Fig. A.4. F110W mag of the PHAT-matched PNe plotted against its F110W-F160W colour. The "likely", "possible", and "unlikely" PNe are shown in blue, yellow, and red, respectively. The PNe previously found by M06 are ringed in black.

detected on the background image and their distribution as a function of magnitude would give us an indication of the number of spurious sources present in our PNe catalogue. However, subjecting these sources to the PNe selection criteria described in Sects. 3.3 and 3.4, we find that even if these detected sources survive the colour selection, they would be rejected as PNe in the point-like selection as their half-light radius is much smaller than that expected from the simulated PNe population.

\section{Appendix B: PNe counterparts in PHAT}

\section{B.1. The M06 PNe counterparts in PHAT}

For each M06 PNe, Veyette et al. (2014) searched for counterparts within $3^{\prime \prime}$. They expected a strong relation between the M06 $m_{5007}$ mag and the PHAT F475W magnitude since the majority of F475W flux is due to the [O III] $5007 \AA$ line, and a colour excess between the PHAT F475W and F814W filters, since PNe are not expected to show a strong continuum excess. Additionally, they utilised the separation between the M06 PNe and the PHAT counterpart, and the roundness and sharpness of its PSF. Of the $711 \mathrm{PNe}$ in the M06 catalogue that overlap the PHAT images, they found that only 467 had counterparts consis-

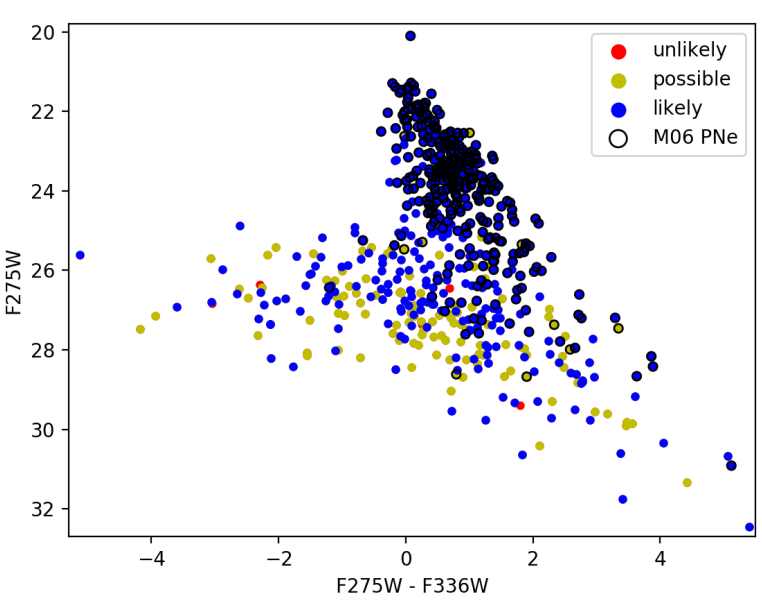

Fig. A.5. F275W mag of the PHAT-matched PNe plotted against its F275W-F336W colour. The "likely", "possible", and "unlikely" PNe are shown in blue, yellow, and red, respectively. The PNe previously found by M06 are ringed in black.

tent with being PNe. The rest were either $\mathrm{H}_{\text {II }}$ regions or possible stellar sources with no PHAT counterpart consistent with being a PNe.

\section{B.2. Further characteristics of the PHAT-matched PNe}

We also check the PNLF for consistency both near the crowded centre (within $0.3 \mathrm{deg}$ ) and in the less crowded outer disc (outside $0.3 \mathrm{deg}$ ) as shown in Fig. A.3. The PNLF is similar in both cases, indicating again that the rise in the PNLF does not depend on crowding, but the value in the less crowded disc shows a more pronounced dip in the PNLF. This may be due to the presence of the $10 \mathrm{kpc}$ star-forming ring in M31 (Barmby et al. 2006), which may be populating the brightest $2.5 \mathrm{mag}$ of the PNLF with PNe evolved from young massive stars. There are $954 \mathrm{PNe}$ that have detections in the PHAT NIR filters. Figure A.4 shows the F110W mag of the PHAT-matched PNe plotted against its F110W-F160W colour. There are 644 PNe that have detections in the PHAT UV filters. Figure A.5 shows the F275W mag of the PHAT-matched PNe plotted against its F275W-F336W colour. The trends in the UV and the NIR are similar to those found by Veyette et al. (2014) for the M06 PNe. 